\title{
Spatial interference shaping for underlay MIMO cognitive networks
}

\author{
Christian Lameiro ${ }^{\mathrm{a}, 1, *}$, Wolfgang Utschick ${ }^{\mathrm{b}}$, Ignacio Santamaría ${ }^{\mathrm{a}, 2}$ \\ ${ }^{a}$ Department of Communications Engineering, Universidad de Cantabria, Spain \\ ${ }^{b}$ Fachgebiet Methoden der Signalverarbeitung, Technische Universität München, Germany
}

\begin{abstract}
Interference temperature (IT) is a widely-used approach for protecting primary users (PUs) from the secondary users (SUs) in underlay cognitive radio. However, when multiple antennas are available at the transmitters and receivers, the spatial structure of the interference comes into play, strongly affecting the performance of the primary network. In this work, we propose interference shaping constraints as an alternative to IT-based approaches. Spatial shaping constraints take account of the structure of interference and exploit it in benefit of the secondary network. Moreover, they can be designed dynamically based on the channel conditions and performance requirements of the PUs. We first show that spatial shaping constraints generalize IT, in that the latter can be expressed as a set of isotropic shaping constraints on each interference dimension. Then, we exemplary consider a PU that has a rate requirement, and propose an algorithm for obtaining suitable shaping matrices, which can be easily modified to include primary transmitter cooperation. This algorithm is performed at the primary receiver using only local channel state information. Afterwards, we address the transceiver optimization of the SU, modeled as a multiple-input multiple-output point-to-point link, and provide optimal and suboptimal transmit covariance designs under the proposed shaping constraints.
\end{abstract}

Keywords: Underlay cognitive radio; interference temperature; spatial shaping; transmitter optimization

\footnotetext{
* Corresponding author

Email addresses: christian. lameiro@sst. upb. de (Christian Lameiro), ut schick atum. de (Wolfgang Utschick), i.santamaria@unican. es (Ignacio Santamaría)

${ }^{1}$ Present address: Signal and System Theory Group, Universität Paderborn, Germany

${ }^{2}$ EURASIP member
} 


\section{Introduction}

The number of wireless devices has significantly increased in the last few years. However, current wireless networks are based on fixed spectrum assignment policies, which make the frequency spectrum unoccupied in many cases and, consequently, results in a poor utilization of

5 the radio resources [1]. In pursuit of an efficient use of them, a flexible usage of the frequency spectrum is needed, in the sense that the channel access is based not only on license holding, but also on its current occupancy [2]. In this context, cognitive radio (CR) has emerged as a promising approach to realize spectrum sharing [3]. CR is based upon the deployment of cognitive users that are capable of accessing the spectrum without disrupting other users communication, 10 by making intelligent use of side information.

$\mathrm{CR}$ is typically applied on a hierarchical spectrum usage, where there are users who have license to access the spectrum, but there are also cognitive users that are able to detect the socalled spectrum holes to opportunistically access the wireless channel when it is not being used by the licensees. The former are usually referred to as primary users (PUs), whereas the latter are typically denoted as secondary users (SUs). This paradigm is called interweave CR and, while it was the original idea of $\mathrm{CR}$, two other paradigms have been proposed, which require different cognition levels: overlay and underlay. An interesting tutorial on the three CR paradigms can be found in [4]. Both overlay and underlay approaches allow concurrent transmission of PUs and SUs, and their main difference is that, in the former case, the PU message is assumed to be known by the SUs, who exploit this side information to cancel their interference or to relay the PU message. Alternatively, the latter case uses interference constraints to guarantee that the interference power at the PUs is below a tolerable level. In this paper, we focus on the underlay CR paradigm and propose a simple modification which, with a limited exchange of information between the PU and SU networks, allows us to greatly improve the SU performance.

In underlay $\mathrm{CR}$ networks, the interference at the primary receivers due to secondary transmissions may be unpredictable. In order for the PUs to meet their performance requirements and operate satisfactorily, such interference must be properly managed. To this end, the interference temperature (IT) approach has been proposed as a means of measuring and controlling the interference in an underlay CR network [5]. More specifically, IT approaches set an acceptable noise floor threshold such that, whenever the interference plus noise power is below that limit, the SUs can access the medium without disrupting the PUs communication. The optimization 
of the SUs transmissions subject to the aforementioned IT constraints has received a great deal of attention in recent years, and a wide range of scenarios and approaches have been considered [6-16]. A particular case of IT constraints are the null constraints, whereby the IT threshold 35 is set to zero, forcing the SUs to opportunistically utilized the unused PU directions [17]-[20]. Furthermore, [19, 20] propose some cooperation between primary and secondary networks, resulting in a significant performance improvement of the secondary network with little impact on the PU. Cooperative cognitive radio networks have been recently proposed as a different level of cooperation between primary and secondary users [21]. This new paradigm follows the overlay

40 CR principle but includes cooperation between PUs and SUs to improve the performance of both types of users.

While in the case of single-antenna nodes or single-carrier transmissions the achievable rate of the PU is completely determined by the total interference plus noise power, when multiple signal dimensions are available (space, frequency...) this is no longer true, since how the interference is distributed in the receiver space strongly affects its performance. Although IT constraints have been also considered at each receiver antenna or at the receiver subspace (i.e., after the decoding process, which is usually referred to as interference perceived at the primary receiver) [6, 9], they still do not capture the whole spatial structure of interference. In fact, multiple antennas have been mainly exploited at the SU side to improve their performance while satisfying the IT constraint. For example, antenna selection can be performed to allow low-complexity secondary transmitters benefit from spatial diversity [13], whereas transmit precoding allows the SUs to reduce the interference level at the primary receivers [14-16]. However, the IT constraint presents limitations for multiple-antenna systems in that the total interference power at the primary receiver does not absolutely reflect its performance. Because of that, multiple antennas can be exploited not only at the SU side to reduce interference, but also at the PU side to set more suitable interference constraints that capture the spatial features of the interference. Following these lines, some recent works have considered alternatives to IT for MIMO systems. For instance, in [22] the PU channel state information (CSI) is exploited at the SU to optimize its transmit covariance matrix subject to an explicit PU rate constraint, which is shown to outperform the

60 IT approach. Nevertheless, such an approach requires additional cross-information between primary and secondary networks, which reduces the actual net throughput and increases the overall system complexity. Linear matrix inequality constraints (similar as the one considered in this 
paper) have been studied in our previous works [23, 24] and in [25], where authors consider the robust optimization of the PU covariance matrix under different interference constraints. Similar interference constraints have also been considered in the context of cellular networks in [26], where shaping constraints are used to upper-bound the worst-case interference covariance matrix when the interference channels are unknown; and in [27, 28] to efficiently coordinate interference with an underlaying device-to-device communication network. The latter is closely related to underlay cognitive radio since the interference constraint is optimized to ensure a given quality of service. The proposed algorithm aims at maximizing the tolerable interference power, but without exploiting the cross-CSI. A more general scenario is considered in [29], where an uplinkdownlink duality is introduced for minimax problems with linear matrix inequality constraints, which usually appear in robust optimization under worst-case interference.

Motivated by these ideas, in this paper we study alternatives to IT constraints when multiple spatial dimensions are available, with reduced cooperation and limited CSI exchange between primary and secondary networks. We base on the same principle as the aforementioned works on interference shaping [25- 28], but focus on the design of the spatial shaping matrices to efficiently handle interference between networks whose cooperation and CSI is limited, which is exemplary illustrated through the underlay CR paradigm. Notice that, unlike conventional underlay CR, our method requires some cooperation between primary and secondary networks, thereby boosting the performance of the SUs while providing a better protection to PUs. This is motivated by the aforementioned cooperative PU-SU schemes, where the benefit of cooperation has been shown for different CR paradigms (see, e.g. [4][19]-[21] and references therein).

This paper builds up on our previous work in [23, 24]. Here, we extend the design of spa85 tial shaping constraints by presenting a more general optimization framework, where primary transmitter cooperation can easily be introduced. Furthermore, we present a stronger connection between spatial shaping and interference temperature, and provide a detailed analysis on the properties of the proposed interference shaping constraints. We additionally address the optimization of a MIMO SU under a spatial shaping constraint and provide several numerical examples that illustrate the performance of the proposed approach compared to IT and the alternative technique presented in [22].

The rest of the paper is organized as follows. Section 2 provides a general description of the problem and introduces the concept of spatial interference shaping. The design of suitable spatial 


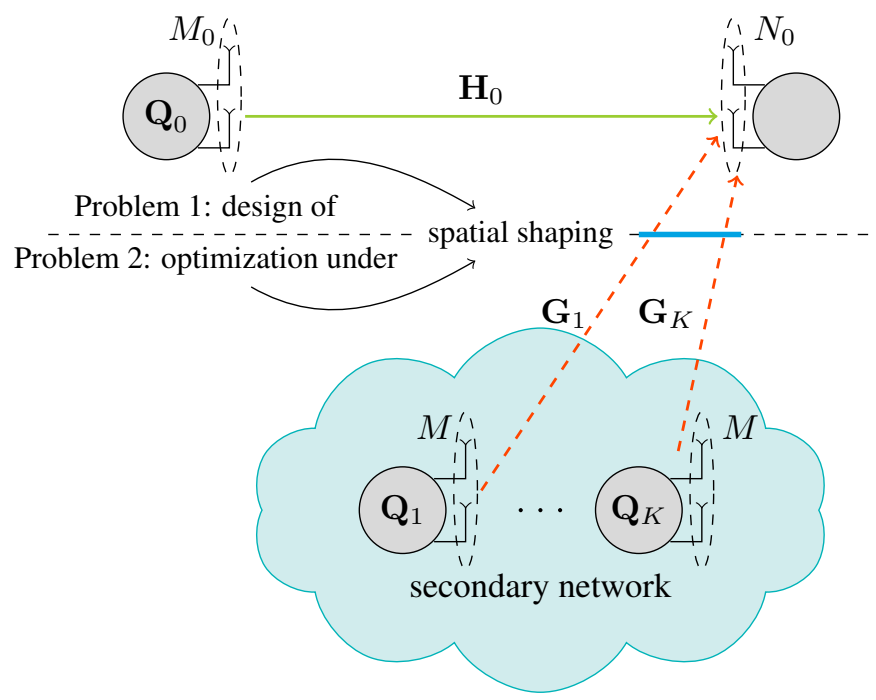

Figure 1: General scenario and problem overview.

shaping constraints is presented in Section 3. In Section 4, we describe the system model from the secondary network standpoint and tackle the optimization of the SU transmission schemes under transmit shaping constraints for a point-to-point MIMO SU. Section[5illustrates the performance of the proposed spatial shaping constraints through several numerical examples. Finally, Section 6 presents the concluding remarks.

\section{General model and problem overview}

\subsection{System description}

Consider a primary point-to-point link where the transmitter and receiver are equipped with $M_{0}$ and $N_{0}$ antennas, respectively. Furthermore, $K$ unlicensed devices or SUs equipped with $M$ transmit antennas each wish also to access the wireless channel, as depicted in Fig. 1. Following the underlay CR paradigm, the SUs are allowed to coexist with the PU as long as they control the generated interference in such a way that a prescribed rate constraint at the PU is guaranteed at the PU. Hence, when the PU is not fully loaded, i.e., its rate requirement is below its point-topoint capacity, the SUs have an opportunity to access the channel by controlling the interference generated at the primary receiver by means of power control or beamforming. 
If we denote the channel from the $k$-th $\mathrm{SU}$ to the primary receiver as $\mathbf{G}_{k}$, and the direct channel of the $\mathrm{PU}$ as $\mathbf{H}_{0}$, the signal received by the latter can be expressed as

$$
\mathbf{y}=\underbrace{\mathbf{H}_{0} \mathbf{x}}_{\text {desired signal }}+\underbrace{\sum_{k=1}^{K} \mathbf{G}_{k} \mathbf{z}_{k}}_{\text {interference from SUs }}+\mathbf{n},
$$

where $\mathbf{x} \sim \mathcal{C N}\left(\mathbf{0}, \mathbf{Q}_{0}\right)$ and $\mathbf{z}_{k} \sim \mathcal{C N}\left(\mathbf{0}, \mathbf{Q}_{k}\right)$ are the transmitted signals by the $\mathrm{PU}$ and $k$ th $\mathrm{SU}$, respectively, with $\mathbf{Q}_{0} \in \mathbb{S}_{+}^{M_{0}}$ and $\mathbf{Q}_{k} \in \mathbb{S}_{+}^{M}$ being the respective transmit covariance matrices $\left(\mathbb{S}_{+}^{N}\right.$ is the set of $N \times N$ positive semidefinite matrices), and $\mathbf{n} \sim \mathcal{C N}\left(\mathbf{0}, \sigma^{2} \mathbf{I}\right)$ is the additive white Gaussian noise (AWGN). Both PU and SU transmit signals are assumed to be independent. Denoting as $P_{0}$ and $P$ the power budgets of the PU and SUs, respectively, the transmit covariance matrices satisfy $\operatorname{Tr}\left(\mathbf{Q}_{0}\right) \leq P_{0}$ and $\operatorname{Tr}\left(\mathbf{Q}_{k}\right) \leq P{ }^{3}$ When the transmit covariance matrix of the $\mathrm{PU}$ is fixed, we can express its achievable rate as a function of the transmit covariance matrices of the SUs as

$$
R_{\mathrm{PU}}\left(\left\{\mathbf{Q}_{k}\right\}_{k=1}^{K}\right)=\log _{2}\left|\mathbf{I}+\left(\sigma^{2} \mathbf{I}+\sum_{k=1}^{K} \mathbf{G}_{k} \mathbf{Q}_{k} \mathbf{G}_{k}^{H}\right)^{-1} \mathbf{H}_{0} \mathbf{Q}_{0} \mathbf{H}_{0}^{H}\right| .
$$

The rate constraint can therefore be written as

$$
R_{\mathrm{PU}}\left(\left\{\mathbf{Q}_{k}\right\}_{k=1}^{K}\right) \geq \bar{R},
$$

where $\bar{R}$ is the prescribed rate requirement.

\subsection{Spatial interference shaping}

There are different approaches that can be carried out to ensure that the PU achieves its rate requirement in spite of the secondary transmissions. In CR, null or little cooperation between primary and secondary networks is typically desirable. Thus, the conventional way consists in setting an interference power constraint, $\operatorname{Tr}\left(\sum_{k=1}^{K} \mathbf{G}_{k} \mathbf{Q}_{k} \mathbf{G}_{k}^{H}\right) \leq t$, where $t$ is the predefined IT threshold, as a surrogate of the rate constraint (3), so that an appropriate selection of $t$ ensures the rate of the PU. This accordingly decouples both networks, as the interference power or IT

\footnotetext{
${ }^{3}$ Without loss of generality, we consider that the secondary transmitters are equipped with the same number of antennas and have equal power budgets. The proposed algorithms can be straightforwardly extended to account for unequal number of antennas and/or power budgets.
} 
constraint bounds the impact of the secondary network over the PU. When the primary receiver has multiple antennas, however, the total interference power does not provide a complete characterization of the interference, since its spatial distribution strongly affects the transmission rate achieved by the PU. With multiantenna systems, the IT threshold, $t$, must be chosen to guarantee a worst-case interference covariance matrix [30]. This can be accomplished by solving the following optimization problem [30]

$$
\begin{aligned}
& \mathcal{P}_{I T}: \underset{t}{\operatorname{maximize}} \quad t, \\
& \text { subject to } \quad \log _{2}\left|\mathbf{I}+\left(\sigma^{2} \mathbf{I}+\mathbf{K}\right)^{-1} \mathbf{H}_{0} \mathbf{Q}_{0} \mathbf{H}_{0}^{H}\right| \geq \bar{R}, \mathbf{K} \in \mathbb{K}(t),
\end{aligned}
$$

where $\mathbb{K}(t)=\{\mathbf{K} \succeq \mathbf{0}: \operatorname{Tr}(\mathbf{K}) \leq t\}$.

As an alternative to IT constraints, in this paper we consider spatial shaping (SS) constraints in order to dynamically exploit the spatial structure of the interference. Unlike IT, the SS approach does not define a specific constraint but a class of constraints. Basically, it consists in limiting or even forbidding interference power in some directions that can be specially detrimental for the PU performance. Spatial shaping constraints can be designed such that particularly harmful spatial structures are avoided, thereby increasing the tolerable interference power without compromising the PU performance. In general terms, the design of the SS constraint can be formulated as a generalization of the IT design problem $\mathcal{P}_{I T}$ as

$$
\begin{aligned}
& \mathcal{P}_{S S}: \underset{\mathbf{S}}{\operatorname{maximize}} f(\mathbf{S}), \\
& \text { subject to } \quad \log _{2}\left|\mathbf{I}+\left(\sigma^{2} \mathbf{I}+\mathbf{K}\right)^{-1} \mathbf{H}_{0} \mathbf{Q}_{0} \mathbf{H}_{0}^{H}\right| \geq \bar{R}, \mathbf{K} \in \mathbb{K}(\mathbf{S}),
\end{aligned}
$$

where the cost function $f(\mathbf{S})$ and the set of admissible interference covariance matrices $\mathbb{K}(\mathbf{S})$ can be designed in many different ways. In this paper, we consider SS constraints of the form 4

$$
\mathbf{Q}_{k} \preceq \mathbf{S}_{k}, k=1, \ldots, K,
$$

where $\mathbf{S}_{k}$ is the positive-semidefinite shaping matrix of the $k$ th SU. By means of (4), the transmit covariance matrix of each secondary transmitter is constrained. In this case, the set of admissible

\footnotetext{
${ }^{4}$ Similar spatial shaping constraints can be considered at different points of the communication link, e.g., by constraining $\mathbf{G}_{k} \mathbf{Q}_{k} \mathbf{G}_{k}^{H}$ or $\sum_{k=1}^{K} \mathbf{G}_{k} \mathbf{Q}_{k} \mathbf{G}_{k}^{H}$, which may require different levels of cooperation and CSI knowledge. In this work, we restrict to spatial shaping of the transmit covariance matrices as in 4 for the sake of exposition.
} 
interference covariance matrix has the form $\mathbb{K}\left(\{\mathbf{S}\}_{k=1}^{K}\right)=\left\{\mathbf{K}: \mathbf{K}=\sum_{k=1}^{K} \mathbf{G}_{k} \mathbf{Q}_{k} \mathbf{G}_{k}^{H}, \mathbf{0} \preceq\right.$ $\left.115 \mathbf{Q}_{k} \preceq \mathbf{S}_{k}\right\}$. The cost function $f\left(\left\{\mathbf{S}_{k}\right\}_{k=1}^{K}\right)$ can be, e.g., $f\left(\left\{\mathbf{S}_{k}\right\}_{k=1}^{K}\right)=\sum_{k=1}^{K} w_{k} \operatorname{Tr}\left(\mathbf{S}_{k}\right)$, which is the weighted sum of allowable transmit powers and is the cost function that we will adopt in this paper; $f\left(\left\{\mathbf{S}_{k}\right\}_{k=1}^{K}\right)=\sum_{k=1}^{K} w_{k} \log _{2}\left|\mathbf{I}+\frac{1}{\sigma^{2}} \mathbf{S}_{k}\right|$, which is an upper-bound of the expected weighted sum-rate of the secondary network; or any other similar concave cost function.

As we will show, the spatial shaping matrices in (4) can be designed to ensure, without applying any additional constraint to the secondary network, the PU rate constraint independently of the specific transmit covariance matrices of the SUs. This means that, similarly to the IT constraint, SS constraints decouple both the primary and secondary networks. Furthermore, since each matrix $\mathbf{S}_{k}$ directly constrains the transmit covariance matrix of the corresponding SU, this approach does not require the SUs to acquire the cross-channel matrices, $\mathbf{G}_{k}$.

In the following lemma, we show that a shaping constraint in the form of (4) can be used as a substitute for the rate constraint (3) by a proper selection of $\mathbf{S}_{k}, k=1, \ldots, K$.

Lemma 1. Let $\mathbf{S}_{k} \in \mathbb{S}_{+}^{N}, k=1, \ldots, K$, be such that $R_{\mathrm{PU}}\left(\left\{\mathbf{S}_{k}\right\}_{k=1}^{K}\right) \geq \bar{R}$. Then $R_{\mathrm{PU}}\left(\left\{\mathbf{Q}_{k}\right\}_{k=1}^{K}\right)$ $\geq \bar{R}$ for all $\mathbf{Q}_{k} \in \mathbb{S}_{+}^{N}$ satisfying $\mathbf{Q}_{k} \preceq \mathbf{S}_{k}$.

Proof. This can easily be proved using the equivalence $\mathbf{X} \preceq \mathbf{Y} \Leftrightarrow \mathbf{X}^{-1} \succeq \mathbf{Y}^{-1}$ [31], which yields

$$
\mathbf{Q}_{k} \preceq \mathbf{S}_{k} \Rightarrow \mathbf{Q}_{\mathbf{H}}^{\frac{H}{2}}\left(\sigma^{2} \mathbf{I}+\sum_{k=1}^{K} \mathbf{G}_{k} \mathbf{Q}_{k} \mathbf{G}_{k}^{H}\right)^{-1} \mathbf{Q}_{\mathbf{H}}^{\frac{1}{2}} \succeq \mathbf{Q}_{\mathbf{H}}^{\frac{H}{2}}\left(\sigma^{2} \mathbf{I}+\sum_{k=1}^{K} \mathbf{G}_{k} \mathbf{S}_{k} \mathbf{G}_{k}^{H}\right)^{-1} \mathbf{Q}_{\mathbf{H}}^{\frac{1}{2}}
$$

where $\mathbf{Q}_{\mathbf{H}}=\mathbf{H}_{0} \mathbf{Q}_{0} \mathbf{H}_{0}^{H}$ and $(\cdot)^{\frac{H}{2}}=\left[(\cdot)^{\frac{1}{2}}\right]^{H}$. By $[5]$, and since $\log _{2}|\mathbf{I}+\mathbf{X}|$ is matrix-monotone

[31], $R_{\mathrm{PU}}\left(\left\{\mathbf{Q}_{k}\right\}_{k=1}^{K}\right) \geq R_{\mathrm{PU}}\left(\left\{\mathbf{S}_{k}\right\}_{k=1}^{K}\right) \geq \bar{R}$ holds, which concludes the proof.

Hence, the rate of the PU is ensured whenever the spatial shaping matrices $\left\{\mathbf{S}_{k}\right\}_{k=1}^{K}$ are designed such that $R_{\mathrm{PU}}\left(\left\{\mathbf{S}_{k}\right\}_{k=1}^{K}\right) \geq \bar{R}$.

\subsection{Problem overview}

There are two different problems addressed in this paper, as illustrated in Fig. 1. In the first one, we take the point of view of the PU and consider the following question: given a rate requirement as in $(3)$, how can we design the spatial shaping matrices $\left\{\mathbf{S}_{k}\right\}_{k=1}^{K}$, such that the performance of the secondary network is maximized, while $(3)$ is fulfilled for all $\mathbf{Q}_{k} \preceq \mathbf{S}_{k}$ ? 
Differently from the IT approach, in which the maximum interference power threshold, $t$, is univocally given by the rate requirement [3] [30], a suitable design of the spatial shaping matrices cooperation with the PU.

- The primary receiver has perfect local CSI, i.e., the channels from each transmitter $\left(\mathbf{H}_{0}\right.$ and $\left.\mathbf{G}_{k}, k=1, \ldots, K\right)$ to the primary receiver are known.

- The primary receiver knows the power budget of the SUs.

- The primary receiver has no additional information about the secondary network, such as its topology or the SU-SU channels.

- The primary transmitter is unaware of the secondary network activity.

Under these assumptions, the primary receiver designs the shaping matrices and feeds them back to the secondary network through a feedback link. At this stage, the PU is concerned with the SU performance only in the design of the shaping constraints. The shaping matrices obtained this way are subsequently used in the second stage by the SUs, where the actual performance of the secondary network is optimized.

In the second problem, we shift attention to the secondary network, and address the transmit covariance matrix optimization under shaping constraints for a point-to-point MIMO SU. Notice that, if the primary and secondary networks were jointly optimized, the whole network could be regarded as a MIMO IC. Although the joint optimization of this IC network topology might be optimal from the point of view of the SUs, it would require a completely-aware and fullycollaborative PU, which clearly departs from the underlay CR paradigm. On the other hand, the proposed approach can be regarded as partially distributed, where first the PU designs the spatial shaping matrices using local CSI and feeds them back to the secondary network, which then exploits this knowledge and its own local CSI to optimize its performance without any further 


\subsection{Spatial shaping versus interference temperature}

Although the SS approach may seem different from IT at first glance, there is a close connection between them. To ensure the instantaneous PU rate, the IT threshold must also be dynamically designed based on the PU rate requirement and its channel matrix. In comparison with the IT approach, the proposed SS designs require only little cooperation increase in that the local SU-PU channels, $\mathbf{G}_{k}, k=1, \ldots, K$, must be estimated by the primary receiver.

From a mathematical viewpoint, IT can actually be shown to admit an SS representation. That is, in general terms, SS encompasses a broader class of constraints that includes IT as a special case. Since the IT constraint affects the trace of the interference covariance matrix $\mathbf{K}$, we have to focus on a shaping constraint on $\mathbf{K}$ in order to show this relationship, i.e., we have to consider the constraint $\mathbf{K} \preceq \mathbf{P}$, where $\mathbf{P}$ is the shaping matrix. Nevertheless, this constraint is implied by $\left\{\mathbf{Q}_{k} \preceq \mathbf{S}_{k}\right\}_{k=1}^{K}$, i.e., $\left\{\mathbf{Q}_{k} \preceq \mathbf{S}_{k}\right\}_{k=1}^{K} \Rightarrow \mathbf{K} \preceq \sum_{k=1}^{K} \mathbf{G}_{k} \mathbf{S}_{k} \mathbf{G}_{k}^{H}=\mathbf{P}$. Now let $\mathbf{K}=\sum_{i=1}^{r} \mathbf{k}_{i} \mathbf{k}_{i}^{H}$, with $r$ being the rank of $\mathbf{K}$, be an arbitrary decomposition, which may represent the aggregation of all incoming interfering streams from the SUs. By introducing the auxiliary shaping matrices $\left\{\mathbf{P}_{i}\right\}_{i=1}^{r}$, the shaping constraint can be written as

$$
\mathbf{K} \preceq \mathbf{P} \Leftrightarrow\left\{\begin{array}{l}
\mathbf{k}_{i} \mathbf{k}_{i}^{H} \preceq \mathbf{P}_{i}, i=1, \ldots, r \\
\sum_{i=1}^{r} \mathbf{P}_{i} \preceq \mathbf{P}
\end{array} .\right.
$$

Therefore, the design of $\mathbf{P}$ following the lines of $\mathcal{P}_{S S}$ can equivalently be accomplished by optimizing the auxiliary shaping matrices $\left\{\mathbf{P}_{i}\right\}_{i=1}^{r}$. Their optimal solution will minimize the uncertainty of the interference and enforce it to match the best case in terms of PU rate. In other words, the optimal matrices $\left\{\mathbf{P}_{i}\right\}_{i=1}^{r}$ will be rank-one since so are $\left\{\mathbf{k}_{i} \mathbf{k}_{i}^{H}\right\}_{i=1}^{r}$, yielding the bidirectional equivalence in (6). On the other hand, when the secondary network is constrained with an IT threshold, we can derive a similar equivalence by using Lemma 3 in Appendix $\mathrm{A}$ and the set of auxiliary IT thresholds $\left\{t_{i}\right\}_{i=1}^{r}$, yielding

$$
\operatorname{Tr}(\mathbf{K}) \leq t \Leftrightarrow\left\{\begin{array}{l}
\mathbf{k}_{i} \mathbf{k}_{i}^{H} \preceq t_{i} \mathbf{I}, i=1, \ldots, r \\
\sum_{i=1}^{r} t_{i} \mathbf{I} \preceq t \mathbf{I}
\end{array} .\right.
$$

By comparing (6) and (7), it can be readily observed that the IT constraint is equivalent to a set of isotropic shaping constraints on each interference dimension, hence presenting a particular case of the spatial shaping (by constraining $\mathbf{P}_{i}$ and $\mathbf{P}$ to be of the form $t_{i} \mathbf{I}$ and $t \mathbf{I}$, respectively). In the IT problem there is a complete uncertainty in the spatial signature of the interference, since the interference directions $\mathbf{k}_{i}$ are not enforced to lie on any particular subspace. 
This relationship shows that the IT constraint admits indeed a specific SS representation. Dropping this particular SS structure leads to more general shaping constraints that permit controlling the spatial signature of the interference. Therefore, although any of the two constraints may serve as a surrogate for the rate constraint (3), spatial interference shaping provides extra degrees of freedom that can be exploited to enhance the secondary network performance by wisely minimizing the uncertainty of the interference.

\section{Design of spatial shaping matrices}

In this section, we address the first problem, i.e., the design of the spatial shaping matrices. Recall that these matrices are computed at the primary receiver exploiting its local CSI, and will be then fed back to the secondary network. Then, the SUs can optimize their own transmit strategy under the constraints provided by the shaping matrices, hence the PU rate is ensured.

\subsection{Admissible transmit power maximization without primary transmitter cooperation}

As previously discussed, the PU has little knowledge about the secondary network. However, in order to compute suitable shaping matrices $\left\{\mathbf{S}_{k}\right\}_{k=1}^{K}$, it must follow some criterion reflecting the performance of the secondary network. In this paper we will use the weighted sum of admissible transmit powers of the SUs as the performance metric since it can be obtained solely through the shaping matrices, hence no additional assumptions are required. Note that increasing the allowable transmit power does not necessarily lead to an increase in the performance of the SUs. For example, the eigenvectors of $\left\{\mathbf{S}_{k}\right\}_{k=1}^{K}$ also affect the performance of the SUs. However, every possible set of eigenvectors is equally a good choice from the PU standpoint due to its lack of knowledge about the secondary network. Consequently, maximizing the allowable transmit power can be considered as a reasonable choice given the considered assumptions. Therefore we propose the following optimization problem as a particular instance of $\mathcal{P}_{S S} 5^{5}$

$$
\begin{array}{ll}
\mathcal{P}_{0}: & \sum_{k=1}^{K} w_{k} \operatorname{Tr}\left(\mathbf{S}_{k}\right), \\
\text { subject to } & R_{\mathrm{PU}}\left(\left\{\mathbf{S}_{k}\right\}_{k=1}^{K}\right) \geq \bar{R}, \\
& \mathbf{0} \preceq \mathbf{S}_{k} \preceq P \mathbf{I}, k=1, \ldots, K .
\end{array}
$$

\footnotetext{
${ }^{5}$ Although we consider the weighted sum of admissible transmit powers as the performance metric, the proposed algorithm (to be described later) is also applicable for any other concave function of $\left\{\mathbf{S}_{k}\right\}_{k=1}^{K}$
} 

the weighted sum of admissible transmit powers (notice that we use the term worst-case to refer to the SS matrices, since they are an upper-bound of the actual transmit covariance matrices). The weights $w_{k}$ can be tuned to achieve fairness among the SUs. For example, users that are farther from the primary receiver will systematically be allowed to transmit more power than users that are closer. Therefore, each weight $w_{k}$ can be selected based on the eigenvalues of $\mathbf{G}_{k}^{H} \mathbf{G}_{k}$, so that fairness can be achieved. We would also like to remark the last constraint in $\mathcal{P}_{0}$, which limits the maximum eigenvalue of $\mathbf{S}_{k}$ by the power budget of the SUs $P$. The rationale behind this constraint is that the SUs are unable to transmit with a signal power higher than $P$ along any spatial direction. Such a constraint may seem counterintuitive at first glance, as the transmit covariance matrix of the $k$ th $\mathrm{SU}$ is also constrained by its power budget as $\operatorname{Tr}\left(\mathbf{Q}_{k}\right) \leq P$. However, it can be noticed that using the constraint $\operatorname{Tr}\left(\mathbf{S}_{k}\right) \leq P$ instead of $\mathbf{S}_{k} \leq P \mathbf{I}$ yields more restrictive shaping matrices in terms of admissible transmit power, i.e., the optimal value of the cost function of $\mathcal{P}_{0}$ is always equal to or greater than that obtained substituting $\mathbf{S}_{k} \preceq P \mathbf{I}$ by $\operatorname{Tr}\left(\mathbf{S}_{k}\right) \leq P$. This is because the set of matrices that fulfill $\operatorname{Tr}\left(\mathbf{S}_{k}\right) \leq P$ constitutes a subset of those satisfying $\mathbf{S}_{k} \preceq P \mathbf{I}$. In addition, $\mathbf{S}_{k}=P \mathbf{I}$ is the least stringent shaping matrix we can choose such that $\mathbf{Q}_{k} \preceq \mathbf{S}_{k}$ is fulfilled for any $\mathbf{Q}_{k}$ satisfying $\operatorname{Tr}\left(\mathbf{Q}_{k}\right) \leq P$. This means that the $\mathrm{SU}$ is actually spatially-unconstrained in that case, which can be the case if the rate constraint of the PU is low, hence admitting any secondary transmission.

$\mathcal{P}_{0}$ is not a convex optimization problem due to the rate constraint, which makes the problem difficult to solve [32]. In [33], an optimization framework for finding Karush-Kuhn-Tucker (KKT) points of non-convex problems was proposed, based on convex approximations of the non-convex constraints. The key idea is to replace the non-convex constraints by a convex approximation at a given point, and solve the resulting problem. Doing this iteratively the method is shown to converge to a KKT point of the original problem (provided that they exist). Let $\tilde{R}_{\mathrm{PU}}\left(\left\{\mathbf{S}_{k}\right\}_{k=1}^{K},\left\{\mathbf{S}_{k}^{\ell}\right\}_{k=1}^{K}\right)$ be the convex approximation of $R_{\mathrm{PU}}\left(\left\{\mathbf{S}_{k}\right\}_{k=1}^{K}\right)$ at $\mathbf{S}_{k}=\mathbf{S}_{k}^{\ell}$,

In words, the solution of $\mathcal{P}_{0}$ is given by the worst-case covariance matrices that maximize $k=1, \ldots, K$. Then, the following properties must be fulfilled for the successive convex approximation method to be applicable to our problem [33]:

1. $R_{\mathrm{PU}}\left(\left\{\mathbf{S}_{k}\right\}_{k=1}^{K}\right) \geq \tilde{R}_{\mathrm{PU}}\left(\left\{\mathbf{S}_{k}\right\}_{k=1}^{K},\left\{\mathbf{S}_{k}^{\ell}\right\}_{k=1}^{K}\right), \forall \mathbf{S}_{k} \in \mathbb{S}_{+}^{N}$.

2. $R_{\mathrm{PU}}\left(\left\{\mathbf{S}_{k}^{\ell}\right\}_{k=1}^{K}\right)=\tilde{R}_{\mathrm{PU}}\left(\left\{\mathbf{S}_{k}^{\ell}\right\}_{k=1}^{K},\left\{\mathbf{S}_{k}^{\ell}\right\}_{k=1}^{K}\right)$.

3. $\nabla_{\mathbf{S}_{k}} R_{\mathrm{PU}}\left(\left\{\mathbf{S}_{k}^{\ell}\right\}_{k=1}^{K}\right)=\nabla_{\mathbf{S}_{k}} \tilde{R}_{\mathrm{PU}}\left(\left\{\mathbf{S}_{k}\right\}_{k=1}^{K},\left\{\mathbf{S}_{k}^{\ell}\right\}_{k=1}^{K}\right), k=1, \ldots, K$, 
where $\nabla_{\mathbf{S}_{k}} R_{\mathrm{PU}}\left(\left\{\mathbf{S}_{k}\right\}_{k=1}^{K}\right)$ is the derivative of $R_{\mathrm{PU}}\left(\left\{\mathbf{S}_{k}\right\}_{k=1}^{K}\right)$ with respect to $\mathbf{S}_{k}$. We first notice that $R_{\mathrm{PU}}\left(\left\{\mathbf{S}_{k}\right\}_{k=1}^{K}\right)$ is convex [34], but should be concave for $\mathcal{P}_{0}$ to be a convex optimization problem. Since the best concave approximation of a convex function is a linear function, we approximate $R_{\mathrm{PU}}\left(\left\{\mathbf{S}_{k}\right\}_{k=1}^{K}\right)$ by a first-order Taylor expansion. It is easy to see that this linear approximation satisfies the above conditions and can thus be used for a successive convex approximation method. Taking this into account, we obtain a sequence of convex approximations of $\mathcal{P}_{0},\left\{\mathcal{P}_{0}^{\ell}\right\}_{\ell=1}^{L}$, where the $\ell$ th approximation is given by

$$
\begin{aligned}
& \mathcal{P}_{0}^{\ell}: \underset{\left\{\mathbf{S}_{k}\right\}_{k=1}^{K}}{\operatorname{maximize}} \sum_{k=1}^{K} w_{k} \operatorname{Tr}\left(\mathbf{S}_{k}\right) \\
& \text { subject to } \quad R_{\mathrm{PU}}\left(\left\{\mathbf{S}_{k}^{\ell-1}\right\}_{k=1}^{K}\right)+\sum_{k=1}^{K} \operatorname{Tr}\left[\nabla_{\mathbf{S}_{k}} R_{\mathrm{PU}}\left(\left\{\mathbf{S}_{k}^{\ell-1}\right\}_{k=1}^{K}\right)^{H}\left(\mathbf{S}_{k}-\mathbf{S}_{k}^{\ell-1}\right)\right] \geq \bar{R},
\end{aligned}
$$

$$
\mathbf{0} \preceq \mathbf{S}_{k} \preceq P \mathbf{I}, k=1, \ldots, K
$$

where $\left\{\mathbf{S}_{k}^{\ell-1}\right\}_{k=1}^{K}$ is set as the optimal solution of $\mathcal{P}_{0}^{\ell-1}$ and $\nabla_{\mathbf{S}_{k}} R_{\mathrm{PU}}\left(\left\{\mathbf{S}_{k}\right\}_{k=1}^{K}\right)$ is given by 91. The proposed successive convex approximation algorithm is summarized in Algorithm 1. Notice that we have to choose an initial point, $\left\{\mathbf{S}_{k}^{\mathrm{init}}\right\}_{k=1}^{K}$, for Algorithm 1 This initial point must satisfy the constraints of the original problem, i.e., $\mathbf{0} \preceq \mathbf{S}_{k}^{\text {init }} \preceq P \mathbf{I}, k=1, \ldots, K$, and ${ }_{225} R_{\mathrm{PU}}\left(\left\{\mathbf{S}_{k}^{\mathrm{init}}\right\}_{k=1}^{K}\right) \geq \bar{R}$. Such an initial point can be found, e.g., by setting $\mathbf{S}_{k}^{\text {init }}=\gamma \mathbf{I}$, being $\gamma$ the highest value in the interval $[0, P]$ such that $R_{\mathrm{PU}}\left(\{\gamma \mathbf{I}\}_{k=1}^{K}\right) \geq \bar{R}$. We would also like to point out that new shaping matrices must be computed by the PU every time its channel or its transmit covariance matrix changes. Nevertheless, this is also the case of any other approach that guarantees the instantaneous rate of the PU. In this regard, the proposed technique seeks a good trade-off between performance and cooperation requirements.

$$
\begin{aligned}
& \nabla_{\mathbf{S}_{k}} R_{\mathrm{PU}}\left(\left\{\mathbf{S}_{k}\right\}_{k=1}^{K}\right)=-\frac{1}{\log 2} \mathbf{G}_{k}^{H}\left(\sigma^{2} \mathbf{I}+\sum_{k=1}^{K} \mathbf{G}_{k} \mathbf{S}_{k} \mathbf{G}_{k}^{H}\right)^{-1} \mathbf{H}_{0} \mathbf{Q}_{0} \mathbf{H}_{0}^{H} \times \\
& {\left[\mathbf{I}+\left(\sigma^{2} \mathbf{I}+\sum_{k=1}^{K} \mathbf{G}_{k} \mathbf{S}_{k} \mathbf{G}_{k}^{H}+\mathbf{H}_{0} \mathbf{Q}_{0} \mathbf{H}_{0}^{H}\right)^{-1} \mathbf{H}_{0} \mathbf{Q}_{0} \mathbf{H}_{0}^{H}\right]\left(\sigma^{2} \mathbf{I}+\sum_{k=1}^{K} \mathbf{G}_{k} \mathbf{S}_{k} \mathbf{G}_{k}^{H}\right)^{-1} \mathbf{G}_{k}}
\end{aligned}
$$


Set $f_{\mathcal{P}_{0}^{0}}^{\star}=0, \ell=0$ and a tolerance, $\epsilon$; where $f_{\mathcal{P}_{0}^{\ell}}^{\star}$ denotes the optimal value of $\mathcal{P}_{0}^{\ell}$

Choose an initial point $\mathbf{S}_{k}^{0}=\mathbf{S}_{k}^{\text {init }}, k=1, \ldots, K$.

repeat

1. $\ell=\ell+1$.

2. Construct $\mathcal{P}_{0}^{\ell}$ by replacing the rate constraint with its first order approximation at $\left\{\mathbf{S}_{k}^{\ell-1}\right\}_{k=1}^{K}$.

3. Solve $\mathcal{P}_{0}^{\ell}$ to obtain $\left\{\mathbf{S}_{k}^{\star}\right\}_{k=1}^{K}$ and $f_{\mathcal{P}_{0}^{\ell}}^{\star}$ and set, for each $k=1, \ldots, K, \mathbf{S}_{k}^{\ell}=\mathbf{S}_{k}^{\star}$.

until $f_{\mathcal{P}_{0}^{\ell}}^{\star}-f_{\mathcal{P}_{0}^{\ell-1}}^{\star} \leq \epsilon$.

Algorithm 1: Successive convex approximation algorithm for finding local optima of $\mathcal{P}_{0}$.

Although the approximated problem $\mathcal{P}_{0}^{\ell}$ is convex and can therefore be solved efficiently by standard numerical methods, it has a special structure that allows us to devise a more focused optimization algorithm with much reduced computational complexity. Since the objective function and approximated rate constraint are linear, the resulting problem at each iteration of Algorithm ${ }_{235} 1$. $\mathcal{P}_{0}^{\ell}$, is a semidefinite programming (SDP) problem, whose optimal solution is characterized by the following lemma.

Lemma 2. Let $\mathbf{S}_{k}^{\star}, k=1, \ldots, K$, be the optimal solution of $\mathcal{P}_{0}^{\ell}$, and let also $\mathbf{S}_{k}^{\star}=\mathbf{F}_{k} \boldsymbol{\Sigma}_{k} \mathbf{F}_{k}^{H}$ and $w_{k}^{-1} \nabla_{\mathbf{S}_{k}} R_{\mathrm{PU}}\left(\left\{\mathbf{S}_{k}^{\ell-1}\right\}_{k=1}^{K}\right)=\mathbf{V}_{k} \boldsymbol{\Lambda}_{k} \mathbf{V}_{k}^{H}$ be the singular value decomposition (SVD). Then $\mathbf{F}_{k}=\mathbf{V}_{k}$. Furthermore, let $\pi(i) \in\{(k, m): 1 \leq k \leq K, 1 \leq m \leq M\}, i=1, \ldots, M K$, with $\pi(i) \neq \pi(j)$ for $i \neq j$, be an ordering such that $\boldsymbol{\Lambda}_{\pi(1)} \leq \boldsymbol{\Lambda}_{\pi(2)} \leq \ldots \leq \boldsymbol{\Lambda}_{\pi(M K)}$, where $\boldsymbol{\Lambda}_{(k, m)}$ is the mth element in the diagonal of $\boldsymbol{\Lambda}_{k}$. Then, the following holds

$$
\boldsymbol{\Sigma}_{\pi(i)}<P \Rightarrow \boldsymbol{\Sigma}_{\pi(i+1)}=0, i=1, \ldots, M K-1 .
$$

Proof. Please refer to Appendix B.

Notice that $\pi(i)$ in Lemma 2 is an index pair, i.e., it takes a value $\pi(i)=\left(k_{i}, m_{i}\right)$, so $\boldsymbol{\Sigma}_{\pi(i)}$ is the $m_{i}$ th element in the diagonal of matrix $\boldsymbol{\Sigma}_{k_{i}}$. First, Lemma 2 states the optimal transmit directions of the SUs in terms of the approximated problem. That is, the eigenvectors of $\nabla_{\mathbf{S}_{k}} R_{\mathrm{PU}}\left(\left\{\mathbf{S}_{k}^{\ell-1}\right\}_{k=1}^{K}\right)^{H}$ associated with the lowest eigenvalues are the directions along which the $k$ th SU interfere the least in terms of the approximated rate expression. Once the optimal transmit directions have been identified, $\mathcal{P}_{0}^{\ell}$ turns into a linear programm (LP), whose solution 


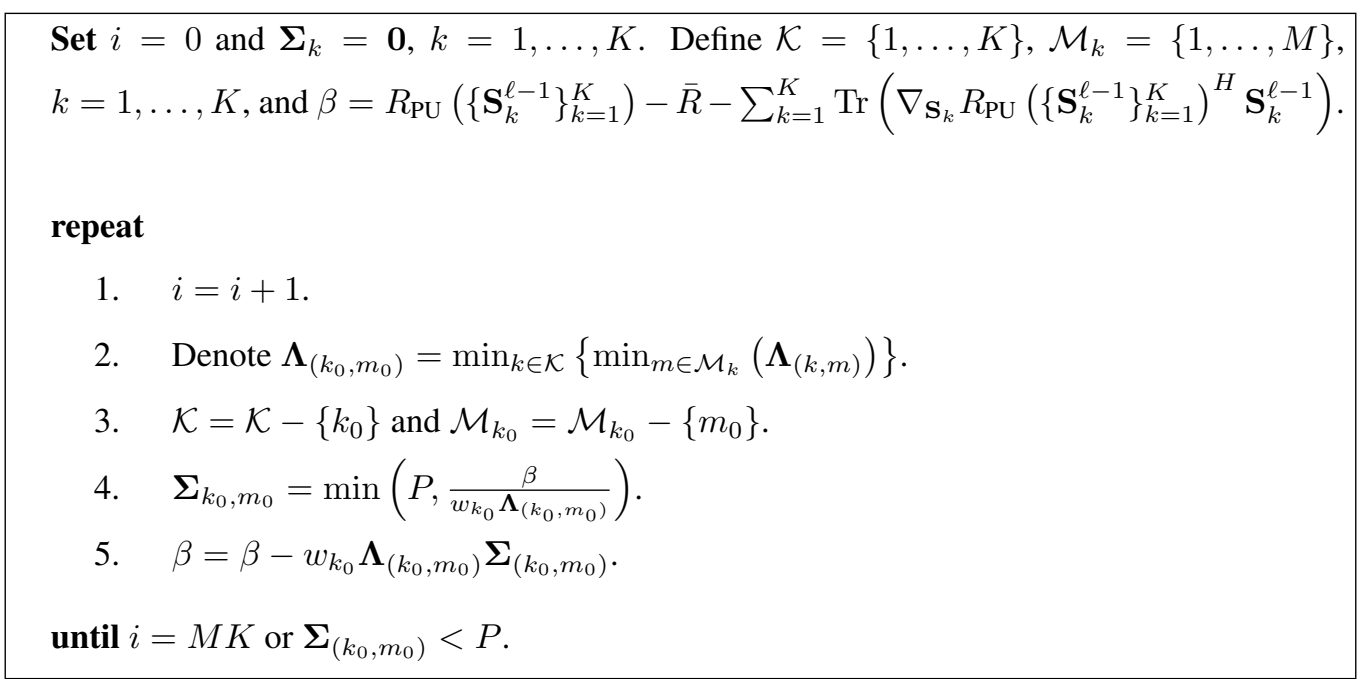

Algorithm 2: Algorithm to find the optimal solution of $\mathcal{P}_{0}^{\ell}$.

is an extreme point of the feasible set. The optimal extreme point is characterized by 10 , which can be interpreted as follows. The secondary network is first allowed to transmit along the relative least harmful direction (i.e., the one associated with $\boldsymbol{\Lambda}_{\pi(1)}$ ) with a signal power such that the approximated rate constraint holds with equality. If such signal power is greater than the SU power budget, it is then set to $P$, what lets the SUs transmit along the next direction (that associated with $\boldsymbol{\Lambda}_{\pi(2)}$ ). This is repeated until the rate constraint holds with equality or all the eigenvalues of $\left\{\mathbf{S}_{k}^{\star}\right\}_{k=1}^{K}$ have been set to $P$. Notice that, in the latter case, $\mathbf{S}_{k}^{\star}=P \mathbf{I}$ holds for $k=1, \ldots, K$. This observation permits computing the optimal solution of $\mathcal{P}_{0}^{\ell}$ in at most $M K$ iterations, as detailed in Algorithm 2

\subsection{Admissible transmit power maximization with primary transmitter cooperation}

So far we have considered that the PU has a fixed transmission strategy, $\mathbf{Q}_{0}$, independent of the shaping matrices $\left\{\mathbf{S}_{k}\right\}_{k=1}^{K}$. However, these matrices upper-bound all possible transmit covariance matrices and, therefore, all possible interference covariance matrices. In other words, $\sum_{k=1}^{K} \mathbf{G}_{k} \mathbf{S}_{k} \mathbf{G}_{k}^{H}$ represents the worst-case interference covariance matrix at the primary receiver. Therefore, it can be exploited at the PU to optimize its transmit covariance matrix so as to further relax the shaping constraint without jeopardizing its data rate. Note that $\mathbf{Q}_{0}$ cannot be directly optimized to reduce the impact of the interference at the secondary network since the PU-SU channels are unknown, and hence the PU transmit covariance matrix can only be opti- 
mized to reduce the stringency of the spatial shaping constraint. To this end, expressing the PU rate given by 22 as a function of both $\left\{\mathbf{S}_{k}\right\}_{k=1}^{K}$ and $\mathbf{Q}_{0}$, i.e., $R_{\mathrm{PU}}\left(\left\{\mathbf{S}_{k}\right\}_{k=1}^{K}, \mathbf{Q}_{0}\right)$, problem $\mathcal{P}_{0}$ can be modified as

$$
\begin{aligned}
& \tilde{\mathcal{P}}_{0}: \operatorname{maximize}_{\left\{\mathbf{S}_{k}\right\}_{k=1}^{K}, \mathbf{Q}_{0}}^{\operatorname{ma}} \quad \sum_{k=1}^{K} w_{k} \operatorname{Tr}\left(\mathbf{S}_{k}\right) \\
& \text { subject to } \quad R_{\mathrm{PU}}\left(\left\{\mathbf{S}_{k}\right\}_{k=1}^{K}, \mathbf{Q}_{0}\right) \geq \bar{R}, \\
& \mathbf{0} \preceq \mathbf{S}_{k} \preceq P \mathbf{I}, k=1, \ldots, K, \\
& \operatorname{Tr}\left(\mathbf{Q}_{0}\right) \leq P_{0}, \\
& \mathbf{Q}_{0} \succeq \mathbf{0}
\end{aligned}
$$

where $P_{0}$ is the power budget of the PU. The joint optimization of the shaping and PU covariance matrices makes $\tilde{\mathcal{P}}_{0}$ even more difficult to solve than the original problem $\mathcal{P}_{0}$. However, we can exploit the iterative nature of the successive convex approximation in Algorithm 1 to include a suboptimal optimization of the transmit covariance matrix $\mathbf{Q}_{0}$ with little increase in complexity. To this end, we first notice that, with $\left\{\mathbf{S}_{k}\right\}_{k=1}^{K}$ being fixed, $\mathbf{Q}_{0}$ must be selected such that the primary user rate is maximized, which translates into the well-known singular value decomposition (SVD) and waterfilling power allocation [35]. Hence, we may perform a joint alternating optimization and successive convex approximation by including an additional transmit optimization step for the PU (SVD and waterfilling) between steps 1 and 2 of Algorithm 1 . Notice that the objective function is still decreasing at each step, so the convergence properties of Algorithm 1 also apply to the joint SS-PU optimization.

\subsection{Discussion}

Before moving to the optimization of the secondary network, we would like to make some remarks about the intuition behind the solution of the proposed algorithms and its connection to the IT approach. To this end, we provide in the following a discussion along with some numerical examples. For all simulations we take $w_{k}=1, k=1, \ldots, K$. We define the transmit signalto-noise ratio $(\mathrm{SNR})$ of the $\mathrm{PU}$ and $\mathrm{SUs}$ as $\mathrm{SNR}_{\mathrm{PU}}=P_{0} / \sigma^{2}$ and $\mathrm{SNR}_{\mathrm{SU}}=P / \sigma^{2}$, respectively, and consider $\sigma^{2}=1$ without loss of generality. Unless stated otherwise, the entries of the channel matrices are independently distributed as complex Gaussian random variables with zero mean and unit variance. All results are obtained by averaging 1000 independent Monte Carlo simulations. 


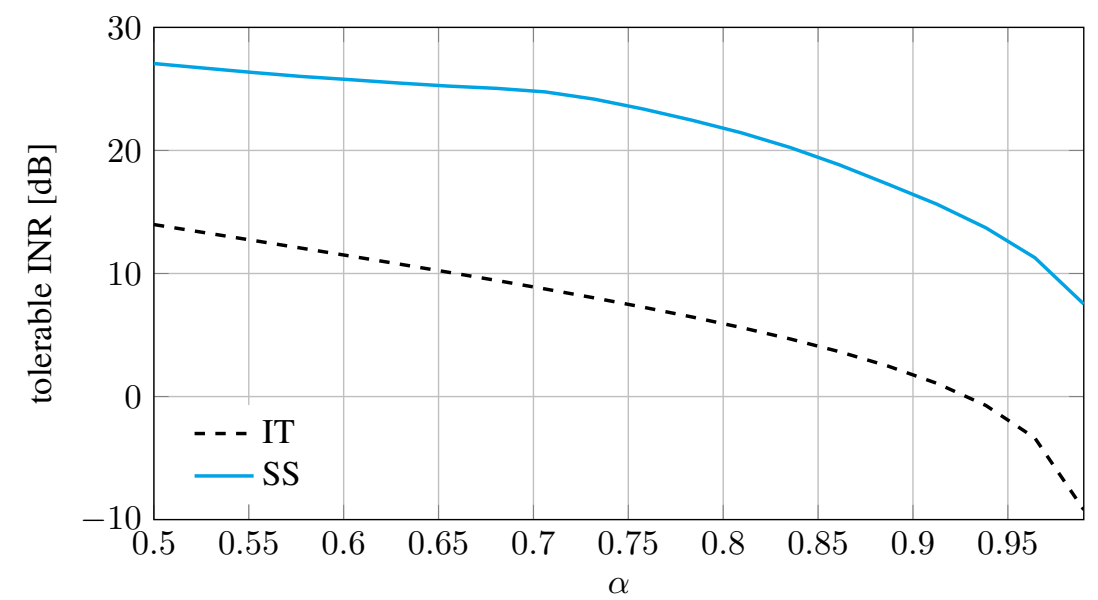

Figure 2: Tolerable INR at the primary receiver for $K=1, M_{0}=N_{0}=M=N=3$, and $\mathrm{SNR}_{\mathrm{SU}}=\mathrm{SNR}_{\mathrm{PU}}=$ $20 \mathrm{~dB}$

\subsubsection{Tolerable interference power}

Since the IT approach only constrains the trace of $\mathbf{K}$, it guarantees the PU a given instantaneous rate by looking at the worst-case interference covariance matrix, and the worst-case interference at each stream is higher the greater the signal power is [30]. In other words, dominant streams are assumed to receive more interference when the IT threshold is obtained, resulting in a very conservative, and therefore pessimistic, interference constraint. On the other hand, the proposed spatial shaping approach works just the other way around. That is, in order to maximize the admissible transmit power of the secondary network, it enforces the SUs to confine their interference along the weakest data streams, so that the impact of such interference is lower and thus the admissible SU transmit power can increase. We illustrate this feature in Fig. 2, which depicts the tolerable interference-to-noise ratio (INR) for $K=1, M_{0}=N_{0}=M=N=3$, and $\mathrm{SNR}_{\mathrm{SU}}=\mathrm{SNR}_{\mathrm{PU}}=20 \mathrm{~dB}$. We also consider that the PU performs the optimal strategy in the absence of interference, i.e., SVD of its direct channel followed by waterfilling power allocation [35], and the rate constraint is taken as a fraction of its maximum achievable rate, i.e., $\bar{R}=\alpha \log _{2}\left|\mathbf{I}+\frac{1}{\sigma^{2}} \mathbf{H}_{0} \mathbf{Q}_{0} \mathbf{H}_{0}^{H}\right|$, where $\alpha \in[0,1]$ is the loading factor. As can be seen in Fig. 2 SS substantially increases the tolerated INR for all values of $\alpha$. 


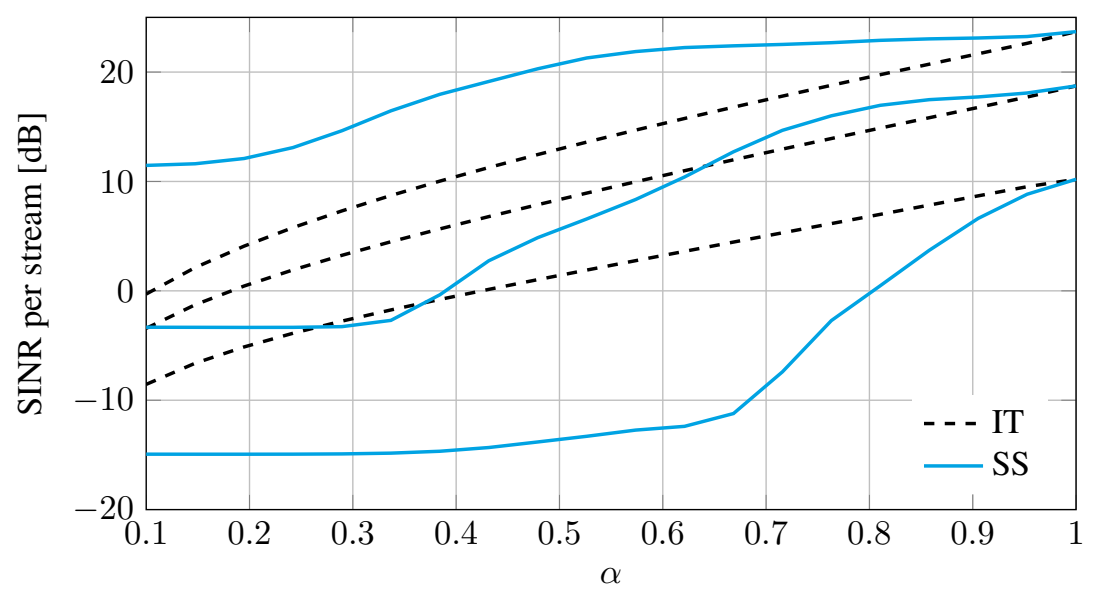

Figure 3: Worst-case SINR at each signal dimension for the same parameters as in Fig. 2

\subsubsection{Dependency with the signal space}

Since in both cases (IT and SS) the interference covariance matrix is upper bounded, it is worth looking at how these upper bounds differ. To this end, let us now consider the dependency between the signal covariance matrix, $\mathbf{Q}_{\mathbf{H}}=\mathbf{H}_{0} \mathbf{Q}_{0} \mathbf{H}_{0}^{H}$, and the interference covariance matrix upper bound, $\mathbf{K}_{\mathrm{UB}}=\sum_{k=1}^{K} \mathbf{G}_{k} \mathbf{S}_{k} \mathbf{G}_{k}^{H}$. We plot the worst-case signal-to-interference-plusnoise ratio (SINR) of each signal dimension of the PU for both IT and SS in Fig. 3 . As can be observed, the proposed spatial shaping approach yields worst-case SINRs that are more spread out than those obtained by the IT approach. Thus, since the strongest signal mode conveys the higher information rate, the SS approach provides it with a minimum SINR that is much higher than that ensured by the IT approach. This is accomplished by lowering the guaranteed SINR at the other signal modes, as they have a lower contribution to the data rate. Notice also that, if we move from $\alpha=1$ to the left part of the figure, the total admissible interference power is increasing. Looking at the SS approach, this has firstly an effect on the SINR of the weakest stream, while the SINR of the remaining two is almost constant, which means that SS is enforcing the interference to be confined into the direction of the weakest stream. If we continue further to the left, the SINR of the second stream starts decreasing as well, while that of the strongest one remains almost unchanged. This is because the interference is being confined into the subspace spanned by the last two signal modes as $\alpha$ decreases. However, the IT approach shows a similar SINR reduction for the three streams, which is the result of not exploiting the spatial dimension. 
As an extreme example, consider the case where $\alpha=1$ but the PU does not use all the available dimensions. That is, there are some signal-free spatial dimensions where the SUs can interfere without reducing the PU rate. Notice that, since $\alpha=1$, the PU does not admit any interference power along signal dimensions. In this case, the conventional IT approach sets an interference power constraint equal to zero, as the worst-case interference covariance matrix assumes interference along the signal dimensions. This could be overcome by constraining the perceived interference power, i.e., the interference power on the signal subspace, rather than the total interference power [9]. This is also referred to as opportunistic interference alignment (IA) [17], where the SUs opportunistically access the channel by confining their transmit signals on the unused receiver subspace, and this is precisely what SS automatically does by optimizing the shaping matrices $\left\{\mathbf{S}_{k}\right\}_{k=1}^{K}$. This can be easily observed by looking at the optimal transmit directions stated in Lemma2. To show this, we rewrite the rate constraint in $\mathcal{P}_{0}^{\ell}$ as

$$
R_{\mathrm{PU}}\left(\left\{\mathbf{S}_{k}^{\ell-1}\right\}_{k=1}^{K}\right)+\operatorname{Tr}\left[\nabla_{\mathbf{K}_{\mathrm{UB}}} R_{\mathrm{PU}}\left(\left\{\mathbf{S}_{k}^{\ell-1}\right\}_{k=1}^{K}\right)^{H} \sum_{k=1}^{K} \mathbf{G}_{k}\left(\mathbf{S}_{k}-\mathbf{S}_{k}^{\ell-1}\right) \mathbf{G}_{k}^{H}\right] \geq \bar{R}
$$

Therefore, if each $\mathbf{G}_{k} \mathbf{S}_{k}^{\ell-1} \mathbf{G}_{k}^{H}$ lies within the null space of $\mathbf{H}_{0} \mathbf{Q}_{0} \mathbf{H}_{0}^{H}$, the smallest eigenvalues of $\nabla_{\mathbf{K}_{\mathrm{UB}}} R_{\mathrm{PU}}\left(\left\{\mathbf{S}_{k}^{\ell-1}\right\}_{k=1}^{K}\right)$ belong to this subspace as well (see $(9)$ ), so $\mathbf{S}_{k}^{\ell}=\mathbf{S}_{k}^{\ell-1}$, i.e., it is a stationary point of $\mathcal{P}_{0}$. As a result, the spatial shaping constraint forces the SUs to align their interference within the unused subspace in an automatic way, clearly showing its superiority over the IT approach.

\subsubsection{Dependency of the interference subspaces}

For multiple secondary users it is worth analyzing how $\mathbf{K}_{k}^{\mathrm{UB}}=\mathbf{G}_{k} \mathbf{S}_{k} \mathbf{G}_{k}^{H}$ and $\mathbf{K}_{i}^{\mathrm{UB}}=$ $\mathbf{G}_{i} \mathbf{S}_{i} \mathbf{G}_{i}^{H}, k \neq i$, relate. As previously discussed, the design of the shaping matrices tends to confine the interference subspace within the weakest signal modes, thus the subspaces spanned by $\mathbf{K}_{k}^{\mathrm{UB}}$ and $\mathbf{K}_{i}^{\mathrm{UB}}$ tend to overlap. In other words, the SUs are enforced to perform IA at the primary receiver. We illustrate this observation in Fig. 4, where the eigenvalues of the normalized interference covariance matrix, which is the covariance matrix divided by the SNR, are depicted as a function of the number of users $K$. The system parameters are $M_{0}=N_{0}=M=N=4$, $\mathrm{SNR}_{\mathrm{SU}}=\mathrm{SNR}_{\mathrm{PU}}=20 \mathrm{~dB}$, and $\alpha=0.7$. Figure 4 shows that, in this scenario, the rank of the interference covariance matrix is two independently of the number of users. This means that the SUs are enforced to perform IA on a specific two-dimensional subspace at the primary 


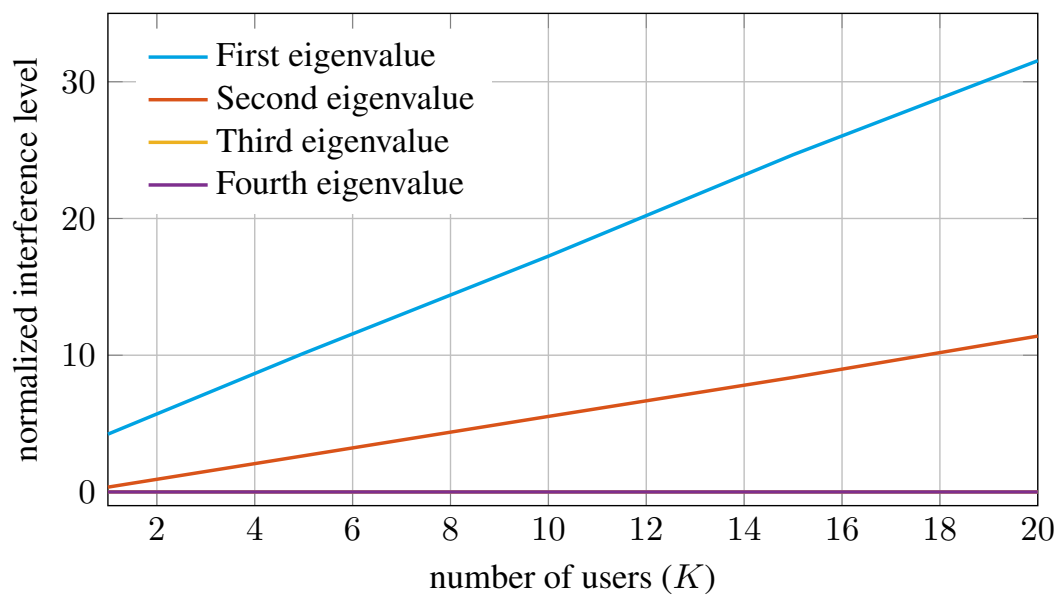

Figure 4: Eigenvalues of the normalized interference covariance matrix for $M_{0}=N_{0}=M=N=4, \mathrm{SNR}_{\mathrm{SU}}=$ $\mathrm{SNR}_{\mathrm{PU}}=20 \mathrm{~dB}$, and $\alpha=0.7$.

receiver. Because of that, the interference level at each of the two dimensions can grow almost linearly with the number of users. Obviously, the rank of the interference covariance matrix is a function of the parameters and channel realizations, so its rank will be different for other scenarios. The key point here is the fact that IA is automatically carried out without requiring the SUs to cooperate with each other if they just design their transmit covariance matrix under the spatial shaping constraint $\left\{\mathbf{Q}_{k} \preceq \mathbf{S}_{k}\right\}_{k=1}^{K}$. That is, IA is achieved at the primary receiver using only local CSI and with non-cooperative SUs. Notice that the latter point is specially interesting when the SUs are low-power devices far from each other, in which case cooperation among them might not be even possible.

\subsubsection{Power allocation of the primary user}

Finally, we provide some intuitions on the solution obtained with the joint SS-PU optimization described in the previous section. The optimal PU transmit covariance matrix is obtained, at each step, as the optimal waterfilling solution given the current shaping matrices. Since the proposed SS tends to allow more interference power into the weakest streams of the PU, the waterfilling step will allocate more power to the strongest streams, which will receive less interference due to the shaping constraint, and less power to the weakest streams, which will receive more interference due to the shaping constraint. Upon convergence, there will be more difference in terms of power allocation and maximum level of interference between the PU streams 


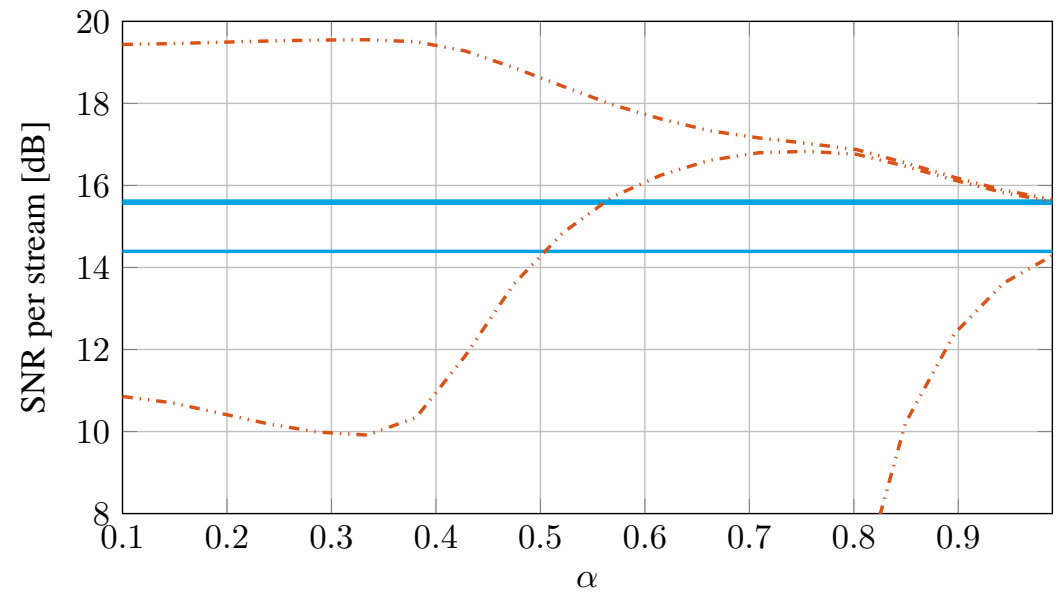

Figure 5: Example of power allocation of the PU data streams for fixed (solid blue lines) and optimized (dashed red lines) transmit covariance matrix. We use the same parameters as in Fig. 2

than with the algorithm without PU optimization, which can even yield a rank-deficient transmit covariance matrix for the PU. Fig. 5 shows an example of the average SNR per stream for both cases, i.e., fixed and optimized PU transmit covariance matrix, which is agreement with our intuitions.

\section{Transmit covariance optimization under shaping constraints}

In this section we address the optimization of a point-to-point MIMO SU under the proposed SS constraint. It is worth stressing that, at this point, there is no need of any further cooperation between networks, or of any joint optimization stage, since the PU is already protected by $\mathbf{Q} \preceq \mathbf{S}$ (see Lemma 11). Consequently, the PU and SU networks are totally decoupled. We consider the following optimization problem 6

$$
\begin{aligned}
& \mathcal{P}_{1}: \underset{\mathbf{Q}}{\operatorname{maximize}} \quad \log _{2}\left|\mathbf{I}+\left(\sigma^{2} \mathbf{I}+\mathbf{G}_{0} \mathbf{Q}_{0} \mathbf{G}_{0}\right)^{-1} \mathbf{H Q} \mathbf{H}^{H}\right|, \\
& \text { subject to } \quad \mathbf{0} \preceq \mathbf{Q} \preceq \mathbf{S} \text {, } \\
& \operatorname{Tr}(\mathbf{Q}) \leq P,
\end{aligned}
$$

where $\mathbf{G}_{0}$ is the PU-SU channel. Although the above problem is convex, we can derive insightful closed-form expressions for some special cases, which we describe in the following proposition.

\footnotetext{
${ }^{6} \mathrm{~A}$ similar problem was considered in [36], but it cannot be applied here due to the transmit power constraint.
} 
Proposition 1. Problem $\mathcal{P}_{1}$ admits a closed-form optimal solution in the following cases

- If $\operatorname{Tr}(\mathbf{S}) \leq P$ :

$$
\mathbf{Q}^{\star}=\mathbf{S}
$$

- If $\operatorname{rank}\left(\mathbf{Q}^{\star}\right)=1$ :

$$
\begin{aligned}
\mathbf{Q}^{\star} & =\mathbf{q}^{\star}\left(\mathbf{q}^{\star}\right)^{H}, \\
\mathbf{q}^{\star} & =\mathbf{S}^{\frac{1}{2}} \nu_{\max }\left[\mathbf{S}^{\frac{1}{2}} \mathbf{H}^{H}\left(\sigma^{2} \mathbf{I}+\mathbf{G}_{0} \mathbf{Q}_{0} \mathbf{G}_{0}\right)^{-1} \mathbf{H S}^{\frac{1}{2}}\right] .
\end{aligned}
$$

- If $\operatorname{rank}\left(\mathbf{Q}^{\star}\right)=\operatorname{rank}(\mathbf{S})$ :

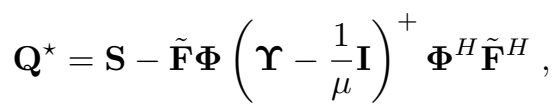

where $\tilde{\mathbf{F}}$ is a unitary basis for the complementary subspace of the nullspace of $\mathbf{S}$, $\tilde{\boldsymbol{\Sigma}}+\left[\tilde{\mathbf{F}}^{H} \mathbf{H}^{H}\left(\sigma^{2} \mathbf{I}+\mathbf{G}_{0} \mathbf{Q}_{0} \mathbf{G}_{0}\right)^{-1} \mathbf{H} \tilde{\mathbf{F}}\right]^{-1}=\boldsymbol{\Phi} \Upsilon \boldsymbol{\Phi}^{H}(S V D)$, and $\mu$ such that $\operatorname{Tr}\left(\mathbf{Q}^{\star}\right)=$ $P$.

\section{Proof. Please refer to Appendix C}

Note that, although the last two cases in Proposition 1 require the rank of the optimal solution, which is unknown, (13)-(15) can still be exploited as follows. First, we can apply (15) to obtain a candidate covariance matrix $\mathbf{Q}^{\prime}$. According to the proof in Appendix C, (15) is obtained assuming that constraint $\mathbf{Q} \succeq \mathbf{0}$ is not active. Hence, if $\mathbf{Q}^{\prime} \succeq \mathbf{0}$ holds, it means that $\mathbf{Q}^{\prime}$ is indeed the optimal solution of $\mathcal{P}_{1}$. Otherwise, the rank of the optimal covariance matrix is lower than the rank of $\mathbf{S}$. In this case we can follow two different approaches. In the first one, we find the optimal solution $\mathbf{Q}^{\star}$ by numerically solving $\mathcal{P}_{1}$. In the second one, we find a suboptimal solution as follows. First, choose the maximum value of $\mu$ such that $(15)$ returns a positive semidefinite matrix, i.e., $\mathbf{Q}^{\prime} \succeq \mathbf{0}$. Second, since this solution violates the power constraint, we scale $\mathbf{Q}^{\prime}$ such that $\operatorname{Tr}\left(\mathbf{Q}^{\prime}\right)=P$. Third, compute the optimal rank-one solution using (13). Finally, take the best between both solutions. Notice that, although the last approach yields, in general, a suboptimal solution, the computational complexity is substantially lower than the numerical method.

\section{Numerical examples}

In this sectionwe compare the rate achieved by four different approaches in the considered point-to-point MIMO SU, namely, IT constraint, explicit rate constraint by exploiting PU CSI 


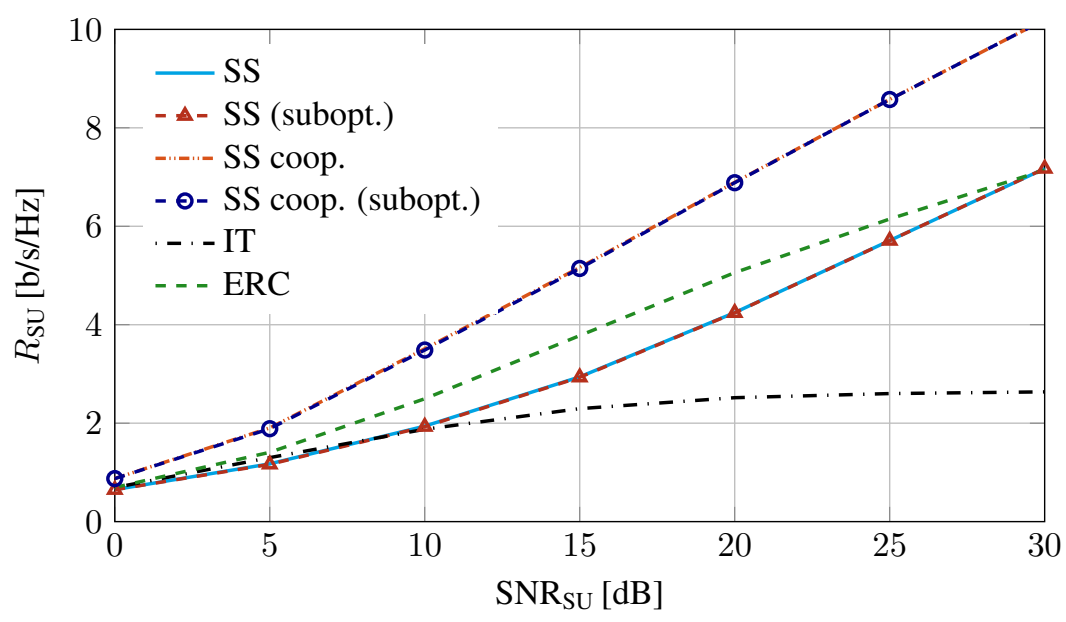

(a) $\alpha=0.75$

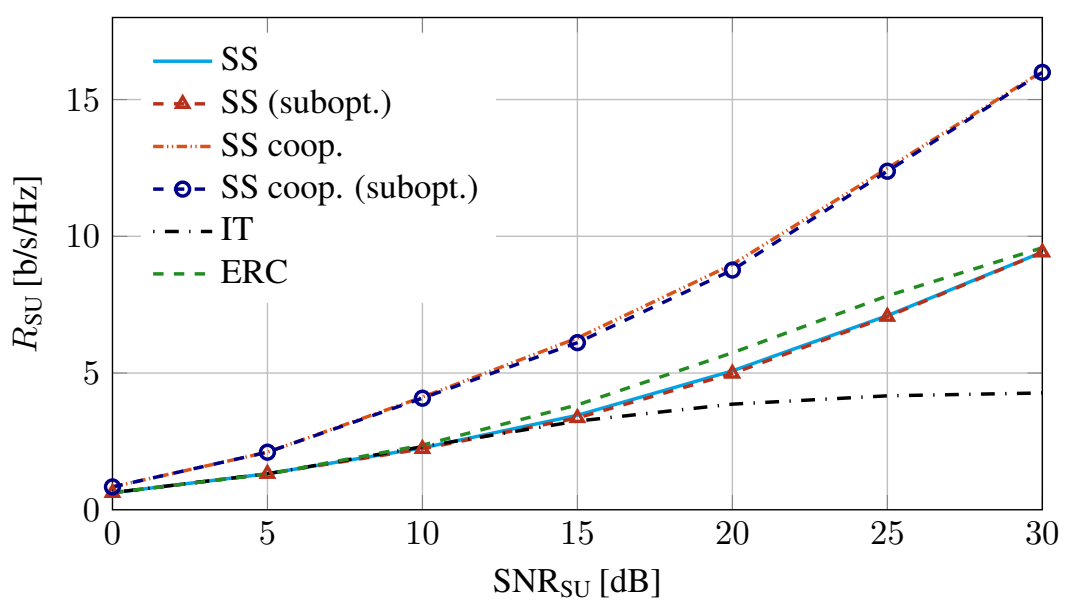

(b) $\alpha=0.5$

Figure 6: Achievable rate of a $3 \times 3 \mathrm{SU}$ coexisting with a $3 \times 3 \mathrm{PU}$ for $\alpha=0.75$ (a) $\alpha=0.5\left[\right.$ (b) and $\mathrm{SNR}_{\mathrm{PU}}=20 \mathrm{~dB}$.

[22] (ERC), and spatial shaping with and without primary transmitter cooperation (SS coop. and SS, respectively). The performance of the suboptimal design of the transmit covariance matrix proposed in the previous section is also depicted for comparison. The number of transmit and receive antennas is 3 for both PU and SU. In the ERC approach, the SU must acquire the SNR of the $\mathrm{PU}, \mathrm{SNR}_{\mathrm{PU}}$, the signal covariance matrix, $\mathbf{H}_{0} \mathbf{Q}_{0} \mathbf{H}_{0}^{H}$, the PU rate requirement, $\bar{R}$, and the SU-PU channel matrix, G. With this information, the transmit covariance matrix of the SU can directly be optimized under the PU rate constraint without the need of interference constraints. Notice, 


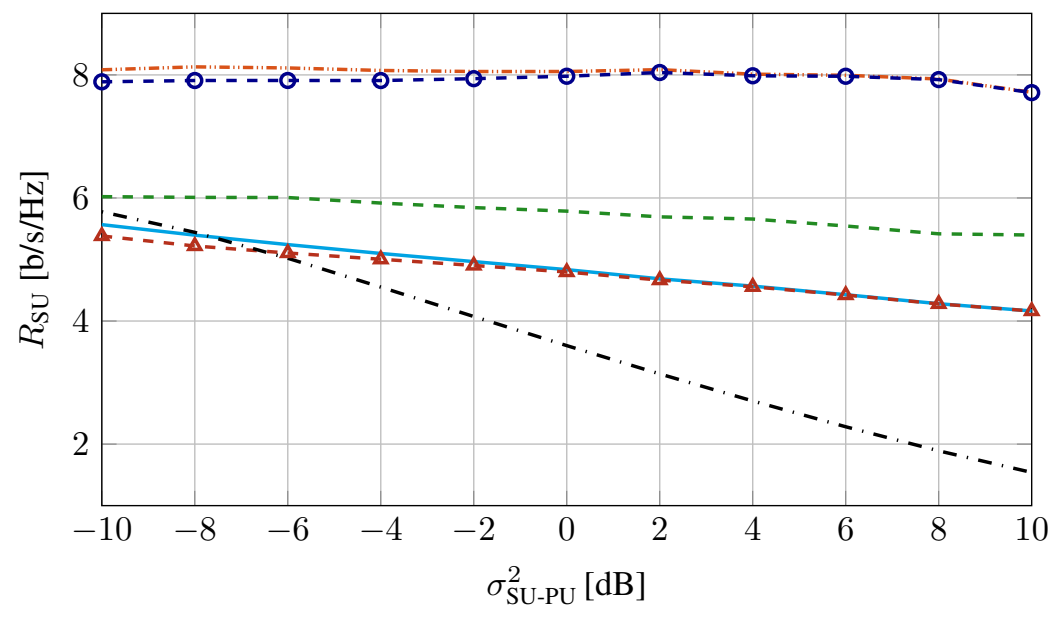

Figure 7: Achievable rate of a $3 \times 3 \mathrm{SU}$ coexisting with a $3 \times 3 \mathrm{PU}$, for $\alpha=0.6$, and $\mathrm{SNR}_{\mathrm{SU}}=\mathrm{SNR}_{\mathrm{PU}}=20 \mathrm{~dB}$. Please refer to the legend of Fig. 6

however, that this approach requires much more signaling and/or cross-information between the PU and the SUs, and can be regarded as fully cooperative. Furthermore, if the secondary network is comprised of several SUs, these must cooperate to satisfy the PU rate constraint, which might not be even possible if they cannot establish a reliable communication, due to, e.g., low transmit power or large distance between them. Figures $6(\mathrm{a})$ and $6(\mathrm{~b})$ show the achievable rate of the SU as a function of its transmit SNR for $\alpha=0.75$ and $\alpha=0.5$, respectively, and $\mathrm{SNR}_{\mathrm{PU}}=20 \mathrm{~dB}$. For low SNR, IT and SS perform similarly, but the latter provides significant improvement as the SNR increases. On the other hand, SS exhibits little degradation with respect to ERC. When the PU optimizes its transmit covariance matrix, the performance of the SU is significantly increased. Notice that optimizing the PU transmit covariance matrix is possible in the proposed approach, but not in the ERC technique. This is a clear advantage of the proposed method, which permits substantially increasing the performance of the SU but keeping at the same time limited cooperation and CSI knowledge. Note that this benefit is not only due to the PU tolerating more interference, but also because the impact of the interference at the $\mathrm{SU}$ is also reduced. The reason is that the PU tends to allocate more power to the directions where the interference is less significant, resulting in a more unequal power allocation that may even end up in a reduction of the rank of the transmit covariance matrix. From the secondary receiver viewpoint, this implies an interference that is stronger in some directions, resulting in a higher achievable rate. 


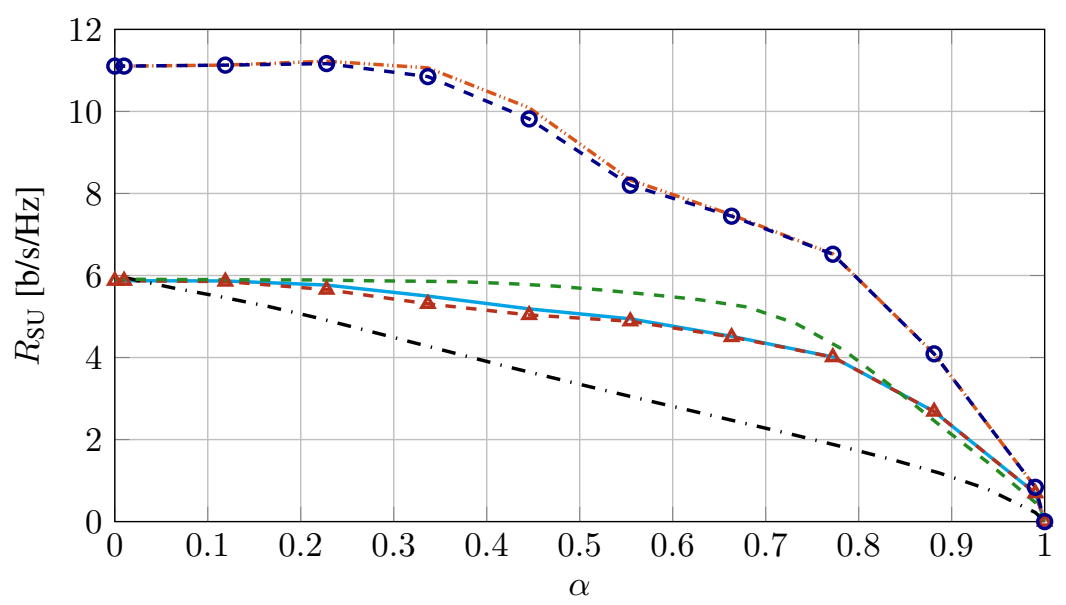

Figure 8: Achievable rate of a $3 \times 3 \mathrm{SU}$ coexisting with a $3 \times 3 \mathrm{PU}$ for $\mathrm{SNR}_{\mathrm{SU}}=\mathrm{SNR}_{\mathrm{PU}}=20 \mathrm{~dB}$. Please refer to the legend of Fig. 6

Figure 7 illustrates the rate of the $\mathrm{SU}$ as a function of $\sigma_{\mathrm{SU}-\mathrm{PU}}^{2}$, which represents the channel strength of the SU-PU link. The other system parameters are set to $\mathrm{SNR}_{\mathrm{SU}}=\mathrm{SNR}_{\mathrm{PU}}=20 \mathrm{~dB}$ and $\alpha=0.6$. It is observed that IT yields a substantial decrease in achievable rate when the interference level increases. On the other hand, SS shows a dependence on $\sigma_{\text {SU-PU }}^{2}$ similar to that of ERC. This is because both techniques exploit the spatial dimension, confining the interference into a reduced dimensional subspace when the interference is more significant. Figure 7 evidences again the limitation of IT to deal with interference in multiple-antenna systems. On the contrary, the proposed approach provides a good trade-off between interference management capabilities and PU-SU cooperation. Furthermore, by letting the primary transmitter adjust its transmit covariance matrix, the performance of the SU greatly increases, substantially outperforming that of ERC and exhibiting an almost constant rate in the considered $\sigma_{\mathrm{SU}-\mathrm{PU}}^{2}$ range.

Finally, we depict in Fig. 8 the dependence of $R_{\mathrm{SU}}$ on $\alpha$ for $\mathrm{SNR}_{\mathrm{SU}}=\mathrm{SNR}_{\mathrm{PU}}=20 \mathrm{~dB}$. Similar conclusions are drawn from this example. A special mention deserves the substantially higher performance of SS coop. for medium and low values of $\alpha$. This observation shows again that the performance increase thanks to the primary transmitter cooperation is mainly due to the fact that the PU tends to reduce the number of transmitted streams, making the interference to the $\mathrm{SU}$ less detrimental. Hence, when $\alpha$ is small, the PU rate constraint is guaranteed with just one data stream, which leaves the secondary receiver with two interference-free dimensions. 
Note that the suboptimal design of the transmit covariance matrix entails a negligible performance loss compared to the optimal solution with the advantage of having much less computational complexity. Note also the SS coop. scheme requires only local CSI at the primary receiver, i.e., the same information as SS. As we have seen in Figs. 66 this scheme yields higher performance than ERC in the considered scenarios, even though ERC requires higher CSI. Furthermore, an optimization of the primary transmitter covariance matrix is not possible with the ERC technique, since the PU does not have any information about the interference.

\section{Conclusion}

In this work, we have considered underlay $\mathrm{CR}$ from two different viewpoints: design of interference shaping constraints for protecting the PUs and optimization of the secondary network subject to these constraints. We have considered multiple-antenna systems, where the interference may come from different spatial directions. Thus, we have first proposed shaping constraints that take the spatial structure of the interference into account and shown that they generalize IT constraints, which pay attention only to the total interference power. Specifically, we have proved that IT constraints can be reformulated as a set of isotropic shaping constraints on each signal dimension. Therefore, the introduction of spatial shaping constraints presents a new way of managing interference in coexisting networks with little cooperation and cross-channel knowledge. By allowing some cooperation with the PU, we proposed an algorithm to obtain suitable shaping matrices when the PU is constrained to achieve a minimum rate requirement. Then, we have addressed the problem of designing the secondary transmit covariance matrix under the proposed shaping constraints for a point-to-point SU. We have shown that the proposed approach substantially improves the rate of the $\mathrm{SU}$ and presents little degradation with respect to approaches that assume global CSI. Furthermore, the proposed technique permits as well optimizing the transmit covariance matrix of the PU taking the interference into account and without requiring additional cooperation or overhead, which significantly boosts the SU performance.

\section{Appendices}

A. Auxiliary lemma

Lemma 3. Let $\mathbf{S} \in \mathbb{S}_{+}^{N}$ and $\mathbf{a} \in \mathbb{C}^{N \times 1}$. Then, if $\mathbf{S}$ is full-rank, aa ${ }^{H} \preceq \mathbf{S}$ holds if and only if $\mathbf{a}^{H} \mathbf{S}^{-1} \mathbf{a} \leq 1$. If $\mathbf{S}$ is rank-deficient, $\mathbf{a a}^{H} \preceq \mathbf{S}$ holds if and only if $\tilde{\mathbf{a}}^{H} \boldsymbol{\Sigma}^{-1} \tilde{\mathbf{a}} \leq 1$, where $\boldsymbol{\Sigma}$ 
is a diagonal matrix containing the non-zero eigenvalues of $\mathbf{S}$ and $\mathbf{a}=\mathbf{F} \tilde{\mathbf{a}}$, with $\mathbf{F}$ being the eigenvectors of $\mathbf{S}$ associated to the non-zero eigenvalues.

${ }_{445}$ Proof. It is easy to see that $\mathbf{a a}{ }^{H} \preceq \mathbf{S}$ is equivalent to $\lambda_{\max }\left[\left(\mathbf{S}^{-\frac{1}{2}}\right)^{H} \mathbf{a a}^{H} \mathbf{S}^{-\frac{1}{2}}\right] \leq 1$ when $\mathbf{S}$ has full rank. Since $\left(\mathbf{S}^{-\frac{1}{2}}\right)^{H} \mathbf{a} \mathbf{a}^{H} \mathbf{S}^{-\frac{1}{2}}$ is rank-one, its maximum eigenvalue is given by $\mathbf{a}^{H} \mathbf{S}^{-1} \mathbf{a}$. When $\mathbf{S}$ is not full-rank, a must lie in the range of $\mathbf{S}$, and can be therefore written as $\mathbf{a}=\mathbf{F} \tilde{\mathbf{a}}$, and the same procedure can be applied, which concludes the proof.

\section{B. Proof of Lemma 2}

Let us denote as $\lambda^{\downarrow}(\mathbf{A})$ and $\lambda^{\uparrow}(\mathbf{A})$ the set of eigenvalues of $\mathbf{A}$ in decreasing and increasing order, respectively. Since $\nabla_{\mathbf{S}_{k}} R_{\mathrm{PU}}\left(\left\{\mathbf{S}_{k}^{\ell-1}\right\}_{k=1}^{K}\right)$ is negative semidefinite (see (9)) we have [37, 9.H.1.h.]

$$
\operatorname{Tr}\left(-\nabla_{\mathbf{S}_{k}} R_{\mathrm{PU}}\left(\left\{\mathbf{S}_{k}^{\ell-1}\right\}_{k=1}^{K}\right)^{H} \mathbf{S}_{k}\right) \geq \sum \lambda^{\downarrow}\left(-\nabla_{\mathbf{S}_{k}} R_{\mathrm{PU}}\left(\left\{\mathbf{S}_{k}^{\ell-1}\right\}_{k=1}^{K}\right)\right) \odot \lambda^{\uparrow}\left(\mathbf{S}_{k}\right)
$$

where $\odot$ denotes Hadamard (element-wise) product, which is equivalent to

$$
\operatorname{Tr}\left(\nabla_{\mathbf{S}_{k}} R_{\mathrm{PU}}\left(\left\{\mathbf{S}_{k}^{\ell-1}\right\}_{k=1}^{K}\right)^{H} \mathbf{S}_{k}\right) \leq \sum \lambda^{\uparrow}\left(\nabla_{\mathbf{S}_{k}} R_{\mathrm{PU}}\left(\left\{\mathbf{S}_{k}^{\ell-1}\right\}_{k=1}^{K}\right)\right) \odot \lambda^{\uparrow}\left(\mathbf{S}_{k}\right)
$$

Since $\sum_{k} \operatorname{Tr}\left(\mathbf{S}_{k}\right)=\sum_{k} \operatorname{Tr}\left(\boldsymbol{\Sigma}_{k}\right)$ and $\mathbf{0} \preceq \mathbf{S}_{k} \preceq P \mathbf{I} \Leftrightarrow \mathbf{0} \preceq \boldsymbol{\Sigma}_{k} \preceq P \mathbf{I}$, the eigenvectors of $\mathbf{S}_{k}$ affect only the constraint $(8)$, which, along with $(\overline{B .2})$, implies $\mathbf{F}_{k}=\mathbf{V}_{k}$ for the optimal solution. Taking this into account, and denoting $\tilde{\mathbf{S}}_{k}=w_{k} \mathbf{S}_{k}=\mathbf{V}_{k} \tilde{\mathbf{\Sigma}}_{k} \mathbf{V}_{k}^{H}$, the cost function of $\mathcal{P}_{0}^{\ell}$ becomes $\sum_{k=1}^{K} \operatorname{Tr}\left(\tilde{\boldsymbol{\Sigma}}_{k}\right)$, and the left-hand side of 8 turns into

$$
\begin{aligned}
& R_{\mathrm{PU}}\left(\left\{\mathbf{S}_{k}^{\ell-1}\right\}_{k=1}^{K}\right)+\sum_{k=1}^{K} \operatorname{Tr}\left[\nabla_{\mathbf{S}_{k}} R_{\mathrm{PU}}\left(\left\{\mathbf{S}_{k}^{\ell-1}\right\}_{k=1}^{K}\right)^{H}\left(\mathbf{S}_{k}-\mathbf{S}_{k}^{\ell-1}\right)\right]= \\
& R_{\mathrm{PU}}\left(\left\{\mathbf{S}_{k}^{\ell-1}\right\}_{k=1}^{K}\right)+\sum_{k=1}^{K} \operatorname{Tr}\left(\nabla_{\mathbf{S}_{k}} R_{\mathrm{PU}}\left(\left\{\mathbf{S}_{k}^{\ell-1}\right\}_{k=1}^{K}\right)^{H} \mathbf{S}_{k}^{\ell-1}\right)-\sum_{k=1}^{K} \sum_{m=1}^{M} \boldsymbol{\Lambda}_{k, m} \tilde{\mathbf{\Sigma}}_{k, m} .
\end{aligned}
$$

Hence, the cost function is maximized by increasing the eigenvalue $\boldsymbol{\Sigma}_{(k, m)}$ of $\mathbf{S}_{k}$, associated with the lowest $\boldsymbol{\Lambda}_{(k, m)}$ until 8 holds with equality or its maximum value $P$ (due to the last constraint in $\mathcal{P}_{0}^{\ell}$ ) is reached. In the latter case there is still room for improvement since (8) does not still hold with equality, thus allowing to increase the next eigenvalue (that associated with the next lowest $\left.\boldsymbol{\Lambda}_{(k, m)}\right)$. Notice that such sequential procedure provides extreme points of the feasible set until the optimum is attained, which yields (10) and concludes the proof. 


\section{Proof of Proposition 1}

In the first case the power constraint is not active, thus the rate is maximized by transmitting the maximum allowable power at each direction, which yields (12). For the second case we make use of Lemma 3 to rewrite $\mathcal{P}_{1}$ as

$$
\tilde{\mathbf{q}}=\arg \max _{\tilde{\mathbf{q}}^{H} \tilde{\boldsymbol{\Sigma}}^{-1} \tilde{\mathbf{q}} \leq 1}\left\{\tilde{\mathbf{q}}^{H} \tilde{\mathbf{F}}^{H} \mathbf{H}^{H}\left(\sigma^{2} \mathbf{I}+\mathbf{G}_{0} \mathbf{Q}_{0} \mathbf{G}_{0}\right)^{-1} \mathbf{H} \tilde{\mathbf{F}} \tilde{\mathbf{q}}\right\}
$$

where $\mathbf{q}=\tilde{\mathbf{F}} \tilde{\mathbf{q}}, \tilde{\mathbf{F}}$ is a matrix containing the eigenvectors of $\mathbf{S}$ with non-zero eigenvalues, and $\tilde{\boldsymbol{\Sigma}}$ is a diagonal matrix with the non-zero eigenvalues of S. Notice that the power constraint, $\mathbf{q}^{H} \mathbf{q} \leq P$, is implicit in $\tilde{\mathbf{q}}^{H} \tilde{\boldsymbol{\Sigma}}^{-1} \tilde{\mathbf{q}} \leq 1$ as long as the actual power budget of the SU is used for the design of the shaping matrix in $\mathcal{P}_{0}$. By letting $\mathbf{a}=\tilde{\mathbf{\Sigma}}^{-\frac{1}{2}} \tilde{\mathbf{q}}$, the foregoing problem turns into the following eigenvalue problem

$$
\mathbf{a}=\arg \max _{\|\mathbf{a}\|=1}\left\{\mathbf{a}^{H} \mathbf{S}^{\frac{1}{2}} \mathbf{H}^{H}\left(\sigma^{2} \mathbf{I}+\mathbf{G}_{0} \mathbf{Q}_{0} \mathbf{G}_{0}\right)^{-1} \mathbf{H} \mathbf{S}^{\frac{1}{2}} \mathbf{a}\right\}
$$

which yields 13 . The solution to the third case can be found by using the slack variable $\mathbf{L}=$ $\mathbf{S}-\mathbf{Q}$. Also, if $\mathbf{S}$ is rank deficient, we may express $\mathbf{Q}=\tilde{\mathbf{F}} \tilde{\mathbf{Q}} \tilde{\mathbf{F}}^{H}$ and $\mathbf{L}=\tilde{\mathbf{F}} \tilde{\mathbf{L}} \tilde{\mathbf{F}}^{H}$. Taking this into account, $\mathcal{P}_{1}$ can be equivalently written as

$$
\begin{array}{ll}
\underset{\tilde{\mathbf{L}}}{\operatorname{maximize}} & \log _{2}\left|\mathbf{I}-\left\{\tilde{\boldsymbol{\Sigma}}+\sigma^{2}\left[\tilde{\mathbf{F}}^{H} \mathbf{H}^{H}\left(\sigma^{2} \mathbf{I}+\mathbf{G}_{0} \mathbf{Q}_{0} \mathbf{G}_{0}\right)^{-1} \mathbf{H} \tilde{\mathbf{F}}\right]^{-1}\right\}^{-1} \tilde{\mathbf{L}}\right|, \\
\text { subject to } & \mathbf{0} \preceq \tilde{\mathbf{L}} \preceq \tilde{\boldsymbol{\Sigma}}, \\
& \operatorname{Tr}(\tilde{\mathbf{L}}) \geq \operatorname{Tr}(\mathbf{S})-P .
\end{array}
$$

Since $\operatorname{rank}\left(\mathbf{Q}^{\star}\right)=\operatorname{rank}(\mathbf{S}), \tilde{\mathbf{L}} \preceq \tilde{\boldsymbol{\Sigma}}$ is not active, which turns problem $\mathbf{C}$.3 into a similar type of the classical MIMO capacity. Hence, the KKT conditions yield the classical solution for $\tilde{\mathbf{L}}$ (i.e., SVD and waterfilling power allocation) but with the reversed sign within the waterfilling. Taking this into account, and since $\mathbf{Q}=\mathbf{S}-\tilde{\mathbf{F}} \tilde{\mathbf{L}} \tilde{\mathbf{F}}^{H}$, we obtain 15 , which concludes the proof.

\section{Acknowledgments}

C. Lameiro and I. Santamaría have received funding from the Spanish Government (MICINN) under projects TEC2013-47141-C4-3-R (RACHEL), TEC2016-75067-C4-4-R (CARMEN) and FPU Grant AP2010-2189. W. Utschick receives financial support from the Deutsche Forschungsgemeinschaft (DFG) under the grant Ut36/15-1. 


\section{References}

[1] S. Haykin, Cognitive radio: brain-empowered wireless communications, IEEE J. Sel. Areas in Commun. 23 (2) (2005) 201-220.

[2] Q. Zhao, B. Sadler, A survey of dynamic spectrum access, IEEE Signal Process. Mag. 24 (3) (2007) $79-89$.

470 [3] J. Mitola, G. Maguire, Cognitive radio: making software radios more personal, IEEE Personal Commun. 6 (4) (1999) 13-18.

[4] A. Goldsmith, S. Jafar, I. Maric, S. Srinivasa, Breaking spectrum gridlock with cognitive radios: An information theoretic perspective, Proc. IEEE 97 (5) (2009) 894-914.

[5] P. J. Kolodzy, Interference temperature: a metric for dynamic spectrum utilization, Int. Journal of Network Management 16 (2) (2006) 103-113.

[6] R. Zhang, Y.-C. Liang, Exploiting multi-antennas for opportunistic spectrum sharing in cognitive radio networks, IEEE J. Sel. Topics in Signal Process. 2 (1) (2008) 88-102.

[7] J.-S. Pang, G. Scutari, D. P. Palomar, F. Facchinei, Design of cognitive radio systems under temperatureinterference constraints: A variational inequality approach, IEEE Trans. Signal Process. 58 (6) (2010) 3251-3271.

[8] G. Scutari, D. Palomar, MIMO cognitive radio: A game theoretical approach, IEEE Trans. Signal Process. 58 (2) (2010) 761-780.

[9] Y. Yang, G. Scutari, P. Song, D. P. Palomar, Robust MIMO cognitive radio systems under interference temperature constraints, IEEE J. Sel. Areas in Commun. 31 (11) (2013) 2465-2482.

[10] E. A. Jorswieck, J. Lv, Spatial shaping in cognitive MIMO MAC with coded legacy transmission, in: Proc. IEEE Int. Workshop Signal Process. Advances Wireless Commun. (SPAWC), San Franciso, CA, USA, 2011, pp. 451455 .

[11] S.-J. Kim, G. B. Giannakis, Optimal resource allocation for MIMO ad hoc cognitive radio networks, IEEE Trans. Inf. Theory 57 (5) (2011) 3117-3131.

[12] L. Zhang, Y.-C. Liang, Y. Xin, Joint beamforming and power allocation for multiple access channels in cognitive radio networks, IEEE J. Sel. Areas in Commun. 26 (1) (2008) 38-51.

[13] R. Sarvendranath, N. B. Mehta, Antenna selection with power adaptation in interference-constrained cognitive radios, IEEE Trans. Commun. 62 (3) (2014) 786-796.

[14] Y. J. Kim, H. J. Lim, M. G. Song, G. H. Im, Power efficient transceiver designs for multi-cell coordination in MIMO cognitive radio networks, IEEE Trans. Commun. 61 (10) (2013) 4127-4138.

[15] T. Luan, X. D. Zhang, F. Gao, J. C. F. Li, M. Lei, Robust interference mitigation against channel uncertainty for MIMO cognitive radios, IEEE Trans. Veh. Technol. 63 (2) (2014) 970-975.

[16] W. Xiong, A. Mukherjee, H. M. Kwon, MIMO cognitive radio user selection with and without primary channel state information, IEEE Trans. Veh. Technol. 65 (2) (2016) 985-991.

[17] S. M. Perlaza, N. Fawaz, S. Lasaulce, M. Debbah, From spectrum pooling to space pooling: Opportunistic interference alignment in MIMO cognitive networks, IEEE Trans. Signal Process. 58 (7) (2010) 3728-3741.

[18] M. Amir, A. El-Keyi, M. Nafie, Constrained interference alignment and the spatial degrees of freedom of MIMO cognitive networks, IEEE Trans. Inf. Theory 57 (5) (2011) 2994-3004.

[19] G. Chen, Z. Xiang, C. Xu, M. Tao, On degrees of freedom of cognitive networks with user cooperation, IEEE Wireless Commun. Lett. 1 (6) (2012) 617-620. 
[20] B. Koo, D. Park, Interference alignment with cooperative primary receiver in cognitive networks, IEEE Commun. Lett. 16 (7) (2012) 1072-1075.

[21] S. Hua, H. Liu, X. Zhuo, M. Wu, S. S. Panwar, Exploiting multiple antennas in cooperative cognitive radio networks, IEEE Trans. Veh. Technol. 63 (7) (2014) 3318-3330.

[22] K. Cumanan, R. Zhang, S. Lambotharan, A new design paradigm for MIMO cognitive radio with primary user rate constraint, IEEE Commun. Lett. 16 (5) (2012) 706-709.

[23] C. Lameiro, W. Utschick, I. Santamaría, Spatial shaping and precoding design for underlay MIMO interference channels, in: Proc. 18th Int. ITG Workshop Smart Antennas (WSA), Erlangen, Germany, 2014, pp. 1-8.

[24] C. Lameiro, I. Santamaría, W. Utschick, Interference shaping constraints for underlay MIMO interference channels, in: Proc. IEEE Int. Conf. Acoustics, Speech and Signal Process. (ICASSP), Florence, Italy, 2014, pp. 1-5.

[25] A. Dotzler, M. Newinger, W. Utschick, Covariance shapes as a cognitive radio concept for receivers with multiple antennas, in: Proc. 19th Int. ITG Workshop Smart Antennas (WSA), Ilmenau, Germany, 2015, pp. 1-8.

[26] A. Dotzler, M. Riemensberger, W. Utschick, G. Dietl, Interference robustness for cellular MIMO networks, in: Proc. IEEE Int. Workshop Signal Process. Advances Wireless Commun. (SPAWC), Darmstadt, Germany, 2012, pp. 229-233.

[27] M. Newinger, A. Dotzler, W. Utschick, Interference shaping for device-to-device communication in cellular networks, in: Proc. IEEE Int. Conf. Commun. (ICC), London, UK, 2015, pp. 4120-4125.

[28] M. Newinger, W. Utschick, Covariance shaping for interference coordination in cellular wireless communication systems, in: Proc. Asilomar Conf. Signals, Syst. and Comput., Pacific Grove, CA, USA, 2015, pp. 648-652.

[29] A. Dotzler, M. Riemensberger, W. Utschick, Minimax duality for MIMO interference networks, Information, Special Issue on Communication Theory 7 (2) (2016) 19

[30] C. Lameiro, W. Utschick, I. Santamaría, Interference-temperature limit for cognitive radio networks with MIMO primary users, in: Proc. Asilomar Conf. Signals, Syst. and Comput., Pacific Grove, CA, USA, 2014, pp. 1-5.

[31] E. Jorswieck, H. Boche, Majorization and matrix-monotone functions in wireless communications, Found. Trends Commun. Inf. Theory 3 (6) (2006) 553-701.

530 [32] S. Boyd, L. Vandenberghe, Convex Optimization, Cambridge University Press, 2004.

[33] B. R. Marks, G. P. Wright, A general inner approximation algorithm for nonconvex mathematical programs, Operations Research 26 (4) (1978) 681-683.

[34] S. Diggavi, T. Cover, The worst additive noise under a covariance constraint, IEEE Trans. Inf. Theory 47 (7) (2001) 3072-3081.

[35] I. E. Telatar, Capacity of multi-antenna Gaussian channels, European Trans. Telecommun. 10 (6) (1999) 585-595.

[36] D. Palomar, Unified framework for linear MIMO transceivers with shaping constraints, IEEE Commun. Lett. 8 (12) (2004) 697-699.

[37] A. W. Marshall, I. Olkin, B. C. Arnold, Inequalities: Theory of Majorization and Its Applications, Springer Series in Statistics, Springer New York, New York, NY, 2011. 